\title{
THE INVERSION OF A GENERAL CLASS OF CONVOLUTION TRANSFORMS $\left(^{1}\right)$
}

\author{
BY \\ I. I. HIRSCHMAN, JR., AND D. V. WIDDER
}

In 1947 , D. V. Widder $\left({ }^{2}\right)$ [18] showed that for a certain class of kernels the convolution transform

$$
f(x)=\int_{-\infty}^{\infty} G(x-t) \phi(t) d t
$$

can be inverted by a linear differential operator of infinite order. The class of kernels was, for convenience, somewhat restricted, and although the Stieltjes transform was included as a special case, the Laplace transform, for example, was not. It is one of the purposes of the present paper to extend the general theory so as to remedy this deficiency. A more significant improvement is that while it was formerly necessary to suppose $\phi(t)$ bounded or absolutely integrable for $(-\infty<t<\infty)$, there are now no restrictions on $\phi(t)$ except those implicitly imposed by the convergence of the integral (1).

By way of motivating the following theory, let us begin with a formal operational solution of equation (1) in the special case when it reduced to the Laplace transform

$$
F(x)=\int_{0}^{\infty} e^{-x t} \Phi(t) d t .
$$

Make an exponential change of variable to obtain

$$
F\left(e^{x}\right) e^{x}=\int_{-\infty}^{\infty} e^{-e^{x-t}} e^{x-t} \Phi\left(e^{-t}\right) d t
$$

This has the same form as equation (1) with

$$
f(x)=F\left(e^{x}\right) e^{x}, \quad G(x)=e^{-\theta x} e^{x}, \quad \phi(t)=\Phi\left(e^{-t}\right) .
$$

To solve it let us apply the bilateral Laplace transform and set

$$
f^{*}(s)=\int_{-\infty}^{\infty} e^{-s t} f(t) d t, \quad \phi^{*}(s)=\int_{-\infty}^{\infty} e^{-s t} \phi(t) d t .
$$

Since the integral (1) is a bilateral convolution and since the transform of $G(t)$ is $\Gamma(1-s)$, the familiar product theorem for the bilateral Laplace trans-

Presented to the Society, February 28, 1948; received by the editors March 26, 1948.

(1) Research paper done under contract with the Office of Naval Research.

(2) The numbers in brackets refer to the bibliography at the end of the paper. 
form gives us

$$
f^{*}(s)=\Gamma(1-s) \phi^{*}(s) .
$$

Now applying the classical complex inversion formula for the bilateral Laplace transform we obtain the solution

$$
\phi(t)=\frac{1}{2 \pi i} \int_{-i \infty}^{i \infty} e^{s t} f^{*}(s) \frac{1}{\Gamma(1-s)} d s .
$$

The same formula also gives

$$
f(t)=\frac{1}{2 \pi i} \int_{-i \infty}^{i \infty} e^{s t} f^{*}(s) d s
$$

Observe that

$$
\begin{aligned}
D f(t) & =f^{\prime}(t)=\frac{1}{2 \pi i} \int_{-i \infty}^{i \infty} e^{s t} s f^{*}(s) d s \\
e^{a D} f(t) & =f(t+a)=\frac{1}{2 \pi i} \int_{-i \infty}^{i \infty} e^{s t} e^{u s} f^{*}(s) d s
\end{aligned}
$$

Hence if we set

$$
P_{n}(s)=\left(1-\frac{s}{n}\right) \prod_{1}^{n-1}\left(1-\frac{s}{k}\right)\left(1+\frac{1}{k}\right)^{*}
$$

successive applications of the operations (5) and (6) give

$$
P_{n}(D) f(t)=\frac{1}{2 \pi i} \int_{-i \infty}^{i \infty} e^{s t} P_{n}(s) f^{*}(s) d s .
$$

But $\boldsymbol{P}_{n}(s)$ tends to $[\Gamma(1-s)]^{-1}$ as $n \rightarrow \infty$. Hence we have formally, by equation (4), that

$$
\lim _{n \rightarrow \infty} P_{n}(D) f(t)=\phi(t) .
$$

Let us now compute the operator $P_{n}(D)$ when the transform is again reduced to its original form (2). The following calculations are immediate.

$$
\begin{gathered}
\left(1-\frac{D}{1}\right) e^{x} F\left(e^{x}\right)=-e^{2 x} F^{\prime}\left(e^{x}\right), \\
\left(1-\frac{D}{1}\right)\left(1-\frac{D}{2}\right) e^{x} F\left(e^{x}\right)=+\frac{1}{2} e^{3 x} F^{\prime \prime}\left(e^{x}\right), \\
\left.P_{n}(D) e^{x} F\left(e^{x}\right)\right|_{x=-u}=\left.\frac{(-1)^{n}}{n !} e^{(n+1) x} F(n)\left(e^{x}\right)\right|_{x=-u+\text { lue n }} .
\end{gathered}
$$


Thus equation (7) becomes

$$
\lim _{n \rightarrow \infty} \frac{(-1)^{n}}{n !}\left(\frac{n}{u}\right)^{n+1} F^{(n)}\left(\frac{n}{u}\right)=\Phi(u) .
$$

But this is precisely the Post-Widder inversion formula, see [14]. The success of the above solution in this special case suggests procedure for the general case. We replace $1 / \Gamma(1-s)$ by a general entire function $E(s)$ with real zeros $a_{k}$ subject to the unique restriction that $\sum_{1}^{\infty} a_{\mathbf{k}}^{-2}<\infty$,

$$
E(s)=e^{b s} \prod_{1}^{\infty}\left(1-\frac{s}{a_{k}}\right) e^{s / a_{k}}
$$

We show directly that $1 / E(s)$ is a bilateral Laplace transform. The same result could be obtained by use of the totally positive functions of Schoenberg [13]. As a consequence we may define the kernel $G(t)$ of equation (1) as

$$
G(t)=\frac{1}{2 \pi i} \int_{-i \infty}^{i \infty} \frac{e^{s t}}{E(s)} d s .
$$

We set

$$
P_{n}(s)=e^{\left(b-b_{n}\right)} \cdot \prod_{k=1}^{n}\left(1-\frac{s}{a_{k}}\right) e^{s / a_{k}},
$$

where $b_{n}$ tends to zero with $1 / n$. We are then able to show that

$$
E(D) f(x)=\lim _{n \rightarrow \infty} P_{n}(D) f(x)=\phi(x),
$$

at least at points of continuity of $\phi(x)$. In order to be assured that the result holds almost everywhere we need to impose further restrictions on the constants $a_{k}$ and $b_{n}$ (see $\$ 25$ ). However, these conditions are satisfied in the cases of the Laplace transform and its iterates. We also treat the more general convolution transform obtained by replacing the integral (1) by a Stieltjes integral. Here again further mild restrictions are needed to obtain our inversion at points of discontinuity of the integrator function. An example is given to show that our results in this direction are, in a certain sense, best possible.

We say that $G(t)$ belongs to class I if there are both positive and negative $a_{k}$. The Stieltjes kernel with $a_{k}= \pm(k-1 / 2), k=1,2, \cdots$, is the prototype of this class. Next $G(t)$ belongs to class II if the $a_{k}$ are all positive (or all negative) and $\sum_{1}^{\infty} a_{k}^{-1}=\infty$. Here the Laplace kernel with $a_{k}=+k, k=1,2, \cdots$, is typical. Finally $G(t)$ belongs to class III if in the previous class the divergent series is replaced by a convergent one. For kernels of class I the region of convergence of the integral (1) is the whole real axis, as is the case for the Stieltjes kernel. For class II the region of convergence is in general a half line, as for the Laplace transform. Kernels of class III may be considered to be 
degenerate. These fundamental differences in behavior make the proofs very different in the three cases, but the final inversion (7) is the same in all cases.

We conclude this introduction by tabulating the form taken by $E(s)$ and $G(t)$ for some of the special cases of our theory. The four most important are

(A) Laplace

$$
\int_{0}^{\infty} e^{-x t} \Phi(t) d t
$$

(B) Stieltjes

$$
\int_{0}^{\infty} \frac{\Phi(t)}{x+t} d t
$$

(C) Iterated Stieltjes $\int_{0}^{\infty} \frac{\log x / t}{x-t} \Phi(t) d t$,

(D) Meijer

$$
\left(\frac{2}{\pi}\right)^{1 / 2} \int_{0}^{\infty}(x t)^{1 / 2} K_{\nu}(x t) \Phi(t) d t .
$$

Here $-1 / 2 \leqq \nu \leqq 1 / 2$ and

$$
K_{\nu}(x)=\frac{\pi}{2} \frac{I_{-\nu}(x)-I_{\nu}(x)}{\sin \pi \nu}, \quad I_{\nu}(x)=\sum_{k=0}^{\infty} \frac{(x / 2)^{2 k+\nu}}{k ! \Gamma(k+\nu+1)} .
$$

For references to these transforms see [14], [15], [5], and [4], respectively. They are subsumed under our theory if for $G(t)$ and $E(s)$ we have

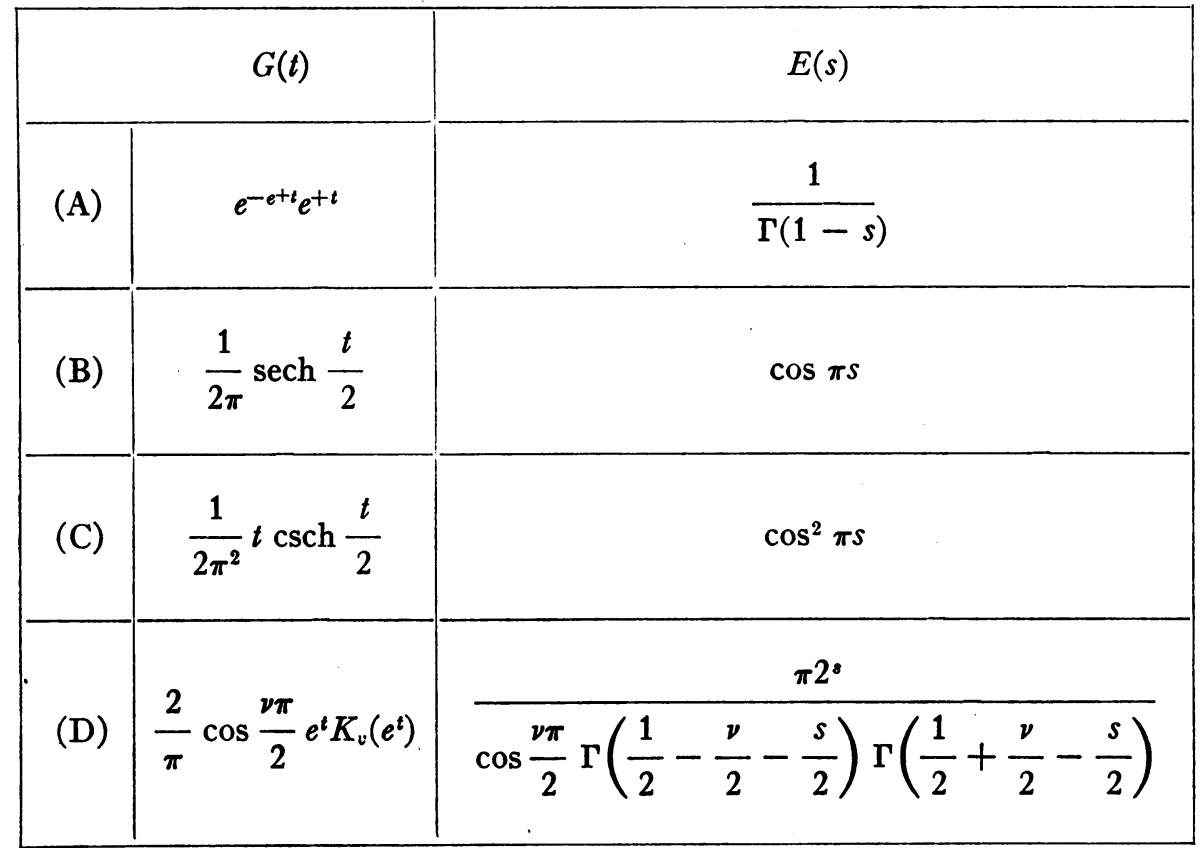


1. The reciprocal of a finite product as a bilateral Laplace transform. Let us make certain definitions, which we shall require here and later. We shall assume throughout that the numbers $\left(b_{m}\right)_{m=0}^{\infty}$ and $\left(b_{m, n}\right)_{m, n=0}^{\infty}$ are real, and that

$$
\begin{aligned}
& \lim _{n \rightarrow \infty} b_{m, n}=b_{m}, \\
& \lim _{m \rightarrow \infty} b_{m}=0 .
\end{aligned}
$$

We shall further suppose that the numbers $\left(a_{k}\right)_{k=1}^{\infty}$ are real, different from zero, and such that the series,

$$
\sum_{k=1}^{\infty} \frac{1}{a_{k}^{2}}
$$

converges. We now define the entire functions

$$
\begin{gathered}
E_{m, n}(s)=e^{b_{m, n} s} \prod_{k=m+1}^{n}\left(1-\frac{s}{a_{k}}\right) e^{s / a_{k}}, \\
E_{m}(s)=e^{b_{m} s} \prod_{k=m+1}^{\infty}\left(1-\frac{s}{a_{k}}\right) e^{s / a_{k}}
\end{gathered}
$$

The convergence of the series (1) insures that the definition of $E_{m}(s)$ has meaning. We shall frequently write $E(s)$ for $E_{0}(s)$ and $b$ for $b_{0}$.

We shall show here that the reciprocal of $E_{m, n}(s)$ is the generating function of a bilateral Laplace transform,

$$
\frac{1}{E_{m, n}(s)}=\int_{-\infty}^{\infty} e^{-s t} G_{m, n}(t) d t,
$$

the integral converging in the largest vertical strip $\left(\alpha_{1}<\sigma<\alpha_{2}\right)$, where $s=\sigma+i \tau$, containing the origin and free of zeros of $E_{m, n}(s)$. The determining function $G_{m, n}(t)$ will be obtained as the convolution of certain elementary functions which we now define.

$$
\begin{array}{ll}
g_{k}(t)=\left\{\begin{array}{cc}
0, & -\infty<t<\frac{1}{a_{k}} \\
-a_{k} e^{a_{k} t-1}, & \frac{1}{a_{k}} \leqq t<\infty
\end{array}\right\} & \text { if } a_{k}<0, \\
g_{k}(t)=\left\{\begin{array}{cc}
a_{k} e^{a_{k} t-1}, & -\infty<t<\frac{1}{a_{k}} \\
0, & \frac{1}{a_{k}} \leqq t<\infty
\end{array}\right\} & \text { if } a_{k}>0 .
\end{array}
$$


THEOREM 1a. If $g_{k}(t)$ is defined by equations (2) then

$$
\int_{-\infty}^{\infty} e^{-s t} g_{k}(t) d t=\left(1-\frac{s}{a_{k}}\right)^{-1} e^{-s / a_{k}}
$$

the integral converging absolutely for $-\infty<\sigma<a_{k}$ when $a_{k}>0$ and for $a_{k}<\sigma<\infty$ when $a_{k}<0$.

By direct computation, $a_{k}>0$, we have

$$
\begin{aligned}
\int_{-\infty}^{\infty} e^{-s t} g_{k}(t) d t & =a_{k} \int_{-\infty}^{1 / a_{k}} e^{-s t} e^{a_{k} t-1} d t \\
& =\left(1-\frac{s}{a_{k}}\right)^{-1} e^{-s / a_{k}}, \quad-\infty<\sigma<a_{k} .
\end{aligned}
$$

We may similarly dispose of the case $a_{k}<0$.

Let us adopt the usual notation

$$
f_{1}(t) * f_{2}(t)=\int_{-\infty}^{\infty} f_{1}(t-u) f_{2}(u) d u
$$

for the bilateral convolution of two functions. We shall require the classical "product theorem" for the product of bilateral transforms $F_{1}(s)$ and $F_{2}(s)$ of $f_{1}(t)$ and $f_{2}(t)$. It asserts that if the transforms converge absolutely in a common region then $F_{1}(s) \cdot F_{2}(s)$ is the transform of the function $(3)$, see $[17 ; \mathrm{pp}$. 248-259]. Using this result we prove the following theorem.

THEOREM 1b. If

$$
\begin{aligned}
\text { 1. } E_{m, n}(s) & =e^{b_{m, n}} \prod_{k=m+1}^{n}\left(1-\frac{s}{a_{k}}\right) e^{s / a_{k}}, \\
\text { 2. } \alpha_{1} & =\max _{a_{k}<0}\left[a_{k},-\infty\right], \alpha_{2}=\min _{a_{k}>0}\left[a_{k},+\infty\right], \\
\text { 3. } G_{m, n}(t) & =g_{m+1} * g_{m+2} * \cdots * g_{n}\left(t-b_{m, n}\right),
\end{aligned}
$$

then

$$
\int_{-\infty}^{\infty} e^{-s t} G_{m, n}(t) d t=\frac{1}{E_{m, n}(s)},
$$

the integral converging absolutely for $\alpha_{1}<\sigma<\alpha_{2}$.

By successive applications of the product theorem to the result of Theorem 1a we obtain

$$
\int_{-\infty}^{\infty} e^{-s t} g_{m+1} * g_{m+2} * \cdots * g_{n}(t) d t=\prod_{k=m+1}^{n}\left(1-\frac{s}{a_{k}}\right)^{-1} e^{-s / a_{k}},
$$


the integral converging absolutely for $\alpha_{1}<\sigma<\alpha_{2}$. On noting that

$$
\int_{-\infty}^{\infty} e^{-s t} f(t-c) d t=e^{-c s} \int_{-\infty}^{\infty} e^{-s t} f(t) d t
$$

our result follows.

COROLlary 1c. If $n-m \geqq 2$, then

$$
G_{m, n}(t)=\frac{1}{2 \pi i} \int_{-i \infty}^{i \infty} \frac{e^{s t}}{E_{m, n}(s)} d s, \quad-\infty<t<\infty,
$$

the integral converging absolutely.

This is a consequence of a classical inversion formula, see $[17 ; \mathrm{pp} .63-80]$. The absolute convergence results from the assumption that $n-m \geqq 2$.

CoROLlaRY 1d. If $n-m \geqq 2$ then $G_{m, n}(t) \in C^{n-m-2}$ for $-\infty<t<\infty$.

This result will be established if we can show that the integrals

$$
\frac{1}{2 \pi i} \int_{-i \infty}^{i \infty} \frac{e^{s t} s^{k}}{E_{m, n}(s)} d s, \quad k=0,1, \cdots, n-m-2,
$$

converge uniformly in $-\infty<t<\infty$. For, then the value of the continuous function (4) will be the $k$ th derivative $G_{m, n}(t)$. The uniform convergence follows from the elementary estimate

$$
\left|E_{m, n}(i \tau)\right| \geqq \frac{|\tau|^{n-m}}{\prod_{k=m+1}^{n}\left|a_{k}\right|}
$$

Corollary 1e. $G_{m, n}(t) \geqq 0(-\infty<t<\infty)$.

This is an immediate consequence of the easily established fact that the convolution of any finite number of non-negative functions is again nonnegative.

2. Inequalities. We wish now to investigate the behavior of the function $E_{m, n}(s)$ and related infinite products as $s$ becomes infinite along vertical lines. Preliminary to this we establish

LEMMA 2a. If

$$
\begin{aligned}
& \text { 1. } E_{m, n}(s)=e^{b_{m, n} s} \prod_{k=m+1}^{n}\left(1-\frac{s}{a_{k}}\right) e^{s / a_{k}}, \\
& \text { 2. } E_{m}(s)=e^{b_{m} s} \prod_{k=m+1}^{\infty}\left(1-\frac{s}{a_{k}}\right) e^{s / a_{k}}
\end{aligned}
$$




$$
\text { 3. } \sum_{k=1}^{\infty} \frac{1}{a_{k}^{2}}<\infty,
$$

then $\left|E_{m, n}(\sigma+i \tau)\right|$ and $\left|E_{m}(\sigma+i \tau)\right|$ are nondecreasing functions of $|\tau|$.

For, if $|\tau| \geqq\left|\tau_{0}\right|$, then

$$
\begin{aligned}
\left|E_{m, n}(\sigma+i \tau)\right| & =e^{b_{m, n} \sigma} \prod_{k=m+1}^{n}\left[\left(1-\frac{\sigma}{a_{k}}\right)^{2}+\left(\frac{\tau}{a_{k}}\right)^{2}\right]^{1 / 2} e^{\sigma / a_{k}} \\
& \geqq e^{b_{m, n} \sigma} \prod_{k=m+1}^{n}\left[\left(1-\frac{\sigma}{a_{k}}\right)^{2}+\left(\frac{\tau_{0}}{a_{k}}\right)^{2}\right]^{1 / 2} e^{\sigma / a_{k}}
\end{aligned}
$$

But the right-hand side of this inequality is $\left|E_{m, n}\left(\sigma+i \tau_{0}\right)\right|$, so that our first conclusion is established. If we allow $n$ to become infinite in (1), we obtain the second conclusion. The resulting infinite products converge by virtue of hypothesis 3 .

THEOREM 2b. If

$$
\begin{aligned}
& \text { 1. } E_{m, n}(s)=e^{b_{m, n} s} \prod_{k=m+1}^{n}\left(1-\frac{s}{a_{k}}\right) e^{s / a_{k}}, \\
& \text { 2. } E_{m}(s)=e^{b_{m} s} \prod_{k=m+1}^{\infty}\left(1-\frac{s}{a_{k}}\right) e^{s / a_{k}} \\
& \text { 3. } \lim _{n \rightarrow \infty} b_{m, n}=b_{m}, \\
& \text { 4. } \sum_{k=1}^{\infty} \frac{1}{a_{k}^{2}}<\infty
\end{aligned}
$$

then for any positive integer $p$

$$
\text { A. } \frac{1}{E_{m, n}(s)}=O\left(|\tau|^{-p}\right), \quad|\tau| \rightarrow \infty,
$$

uniformly for $n \geqq p+m$ and $\sigma$ in any finite interval,

$$
\text { B. } \frac{1}{E_{m}(s)}=O\left(|\tau|^{-p}\right)
$$

uniformly for $\sigma$ in any finite interval.

As in $\$ 1$ we see that for $|\sigma| \leqq R<\infty$

$$
\left|\prod_{k=m+1}^{m+p}\left(1-\frac{\sigma+i \tau}{a_{k}}\right) e^{(\sigma+i \tau) / a_{k}}\right| \geqq A|\tau|^{p},
$$

where 


$$
A=e^{-R C} \prod_{k=m+1}^{m+p} 1 /\left|a_{k}\right|, \quad C=\sum_{k=m+1}^{m+p} 1 /\left|a_{k}\right|
$$

Now choose any $\tau_{0} \neq 0$ and set

$$
\begin{aligned}
B_{m, n}(\sigma) & =\left|e^{b_{m, n}\left(\sigma+i \tau_{0}\right)} \prod_{k=m+p+1}^{n}\left(1-\frac{\sigma+i \tau_{0}}{a_{k}}\right) e^{\left(\sigma+i \tau_{0}\right) / a_{k}}\right|, \\
B_{m}(\sigma) & =e^{b_{m} \sigma} \prod_{k=m+p+1}^{\infty}\left|1-\frac{\sigma+i \tau_{0}}{a_{k}}\right| e^{\sigma / a k}, \\
B_{m, n} & =\min _{|\sigma| \leqq R} B_{m, n}(\sigma), \quad B_{m}=\liminf _{n \rightarrow \infty} B_{m, n} .
\end{aligned}
$$

Then $B_{m} \neq 0$. For, $B_{m}(\sigma) \neq 0$ in $|\sigma| \leqq R$ and has a positive minimum $\delta$ there. Moreover, $B_{m, n}(\sigma)$ tends uniformly to $B_{m}(\sigma)$ in that interval as $n \rightarrow \infty$. Hence there exists $n_{0}$ such that for $n>n_{0}$

$$
\left|B_{m}(\sigma)-B_{m, n}(\sigma)\right|<\delta / 2, \quad|\sigma| \leqq R .
$$

If $B_{m}$ were zero there would exist $n_{1}>n_{0}$ and $\sigma_{0},\left|\sigma_{0}\right| \leqq R$, such that

$$
B_{m, n_{1}}=B_{m, n_{1}}\left(\sigma_{0}\right)<\delta / 2 \text {. }
$$

But this inequality with (2) would imply that $B_{m}\left(\sigma_{0}\right)<\delta$, a contradiction.

It is now evident that there exists a positive constant $B$ such that

$$
B_{m, n}(\sigma) \geqq B, \quad n \geqq m+p,|\sigma| \leqq R .
$$

Then combining this inequality with (1) and making use of Lemma $2 \mathrm{a}$ we obtain $\left|E_{m, n}(s)\right| \geqq A B|\tau|^{p},|\sigma| \leqq R,|\tau| \geqq\left|\tau_{0}\right|$. Since $A B$ is independent of $n$ and $\sigma$ and is different from zero conclusion $A$ is established. Conclusion $B$ follows by letting $n \rightarrow \infty$.

3. The reciprocal of an infinite product as a bilateral Laplace transform. We next extend the result of Theorem $1 \mathrm{~b}$ to apply to the infinite product which defines the entire function $E_{m}(s)$.

Theorem 3a. If

1. $E_{m}(s)=e^{b_{m} s} \prod_{k=m+1}^{\infty}\left(1-\frac{s}{a_{k}}\right) e^{s / a_{k}}$,

2. $\sum_{k=1}^{\infty} \frac{1}{a_{k}^{2}}<\infty$,

3. $\alpha_{1}=\max _{a_{k}<0}\left[a_{k},-\infty\right\rfloor ; \quad \alpha_{2}=\min _{a_{k}>0}\left[a_{k},+\infty\right]$,

4. $G_{m}(t)=\frac{1}{2 \pi i} \int_{-i \infty}^{i \infty} \frac{e^{s t}}{E_{m}(s)} d s$, 
then

$$
\int_{-\infty}^{\infty} e^{-s t} G_{m}(t) d t=\frac{1}{E_{m}(s)},
$$

the integral converging absolutely for $\alpha_{1}<\sigma<\alpha_{2}$. (The subscript $m$ will by convention be dropped when $m=0$.)

For the proof we need the following result of $\mathrm{H}$. Hamburger, see $[17$; pp. 265-267]: If

a. $f(s)$ is analytic for $\alpha_{1}<\sigma<\alpha_{2}$,

b. $\lim _{|\tau| \rightarrow \infty} f(s)=0$ uniformly in every subinterval $\alpha_{1}<\sigma_{1} \leqq \sigma \leqq \sigma_{2}<\alpha_{2}$,

c. $\int_{-\infty}^{\infty}|f(\sigma+i \tau)| d \tau<\infty$ for $\alpha_{1}<\sigma<\alpha_{2}$,

d. $G(t)=\frac{1}{2 \pi i} \int_{c-i \infty}^{c+i \infty} e^{s t} f(s) d s$ for some $c, \alpha_{1}<c<\alpha_{2}$,

then

$$
f(s)=\int_{-\infty}^{\infty} e^{-s t} G(t) d t,
$$

the integral converging absolutely for $\alpha_{1}<\sigma<\alpha_{2}$.

If we set $f(s)=1 / E_{m}(s)$, hypothesis a becomes evident from the definition of $E_{m}(s)$. Hypotheses $\mathrm{b}$ and $\mathrm{c}$ follow from Theorem $2 \mathrm{~b}$. Since $\alpha_{1}<0<\alpha_{2}$, we may choose $c=0$ in the integral $d$. It converges absolutely by Theorem $2 b$. Theorem $3 \mathrm{a}$ is thus established.

Corollary 3b. If $G_{m}(t)$ is defined as in Theorem 3a then $G_{m}(t) \in C^{\infty}$.

For, it is clear from Theorem $2 b$ that for every positive integer $r$ the integral

$$
\frac{1}{2 \pi i} \int_{-i \infty}^{i \infty} \frac{s^{r} e^{s t}}{E_{m}(s)} d s
$$

converges uniformly in every finite interval, $-R \leqq t \leqq R$, and consequently is equal to $G_{m}^{(r)}(t)$.

4. The infinite case as limit of the finite. We now prove that the determining function $G_{m}(t)$ of the previous seztion is the limit of the determining functions $G_{m, n}(t)$ of $\S 1$. It is natural to expect this since the same relation holds between the corresponding generating functions.

Theorem 4a. If $G_{m, n}(t)$ and $G_{m}(t)$ are defined as in Theorem $1 \mathrm{~b}$ and 3a, respectively, then for $r=0,1, \cdots$ 


$$
\lim _{n \rightarrow \infty} G_{m, n}^{(r)}(t)=G_{m}^{(r)}(t),
$$

uniformly for $(-\infty<t<\infty)$.

With $m$ and $r$ fixed choose $n \geqq r+m+2$ so that the integral defining $G_{m, n}^{(r)}(t)$ will converge absolutely. Then

$$
\begin{gathered}
I_{n}=G_{m}^{(r)}(t)-G_{m, n}^{(r)}(t)=\frac{1}{2 \pi i} \int_{-i \infty}^{i \infty} e^{s t} s\left[\frac{1}{E_{m}(s)}-\frac{1}{E_{m, n}(s)}\right] d s \\
\left|I_{n}\right| \leqq \frac{1}{2 \pi} \int_{-\infty}^{\infty}|y| r\left|\frac{1}{E_{m}(i y)}-\frac{1}{E_{m, n}(i y)}\right| d y .
\end{gathered}
$$

Since the integral on the right-hand side of (1) depends in no way on $t$, the uniformity conclusion of the theorem will follow automatically if we show that this integral tends to zero with $1 / n$. Let us now apply Theorem $2 \mathrm{~b}$ with $p=r+2$. There must exist constants $M$ and $y_{0}$, independent of $n$, such that

$$
\left|\frac{1}{E_{m}(i y)}\right| \leqq M|y|^{-p}, \quad\left|\frac{1}{E_{m, n}(i y)}\right| \leqq M|y|^{-p}, \quad|y|>y_{0} .
$$

If $R$ is an arbitrary constant greater than $y_{0}$, it is clear by combining integrals over the ranges $(-\infty,-R)$ and $(R, \infty)$ that

$$
\left|I_{n}\right| \leqq \frac{M}{\pi} \int_{R}^{\infty}|y|^{r-p} d y+\frac{1}{2 \pi} \int_{-R}^{R}|y| r\left|\frac{1}{E_{m}(i y)}-\frac{1}{E_{m, n}(i y)}\right| d y .
$$

But from the general theory of the expansions of entire functions into infinite products the integrand of the second integral on the right tends to zero uniformly in $-R \leqq y \leqq R$ as $n \rightarrow \infty$. Hence

$$
\limsup _{n \rightarrow \infty}\left|I_{n}\right| \leqq \frac{M}{\pi} \int_{R}^{\infty} \frac{d y}{y^{2}} .
$$

Since $R$ can be chosen arbitrarily large, we see that $\lim I_{n}=0$, and the proof is complete.

Corollary 4b. $G_{m}(t) \geqq 0(-\infty<t<\infty), m=0,1,2, \cdots$. This follows from Theorem $4 \mathrm{a}$ and Corollary $1 \mathrm{e}$.

5. Evaluation of residues. In order to shift the path of integration in the integrals

$$
\int_{-i \infty}^{\infty} \frac{e^{s t}}{E_{m, n}(s)} d s, \quad \int_{-i \infty}^{\infty} \frac{e^{s t}}{E_{m}(s)} d s
$$

it will be necessary for us to know the residues of the integrands at the poles. If, by convention, we permit the value $n=\infty$ and set $b_{m, \infty}=b_{m}$, we may avoid repetition by treating both integrals at once. 
THEOREM 5. If $a_{\nu}$ is a zero of $E_{m, n}(s)$ of multiplicity $\mu+1$, then the residue $R_{\nu}$ of $e^{s t} / E_{m, n}(s)$ at the point $s=a_{\nu}$ is

$$
R_{\nu}=e^{a_{\nu} t} P_{\mu}(t),
$$

where $P_{\mu}(t)$ is a real polynomial of degree $\mu$ with coefficient of $t^{\mu}$ equal to

$$
p=\frac{\left(-a_{\nu}\right)^{\mu+1}}{\mu !} \frac{e^{-a_{\nu}}}{\prod_{k=m+1, a_{k} \ngtr a_{\nu}}^{n}\left(1-\frac{a_{\nu}}{a_{k}}\right) e^{a_{\nu} / a_{k}}},
$$

where

$$
c=b_{m, n}+\frac{\mu+1}{a_{v}} .
$$

Since $E_{m, n}(s)$ has a root of order $\mu+1$ at $s=a_{v}$ we have

$$
E_{m, n}(s)=\left(s-a_{v}\right)^{\mu+1} e^{c s} \Omega(s),
$$

where $1 / \Omega(s)$, being analytic at $s=a_{v}$, has a development of the form

$$
\frac{1}{\Omega(s)}=\sum_{j=0}^{\infty} \omega_{j}\left(s-a_{\nu}\right)^{j}
$$

The explicit factorization of $E_{m, n}(s)$ shows that

$$
\omega_{0}=p \mu ! e^{a_{v}} \text {. }
$$

Now

$$
\frac{e^{s t}}{E_{m, n}(s)}=\frac{e^{s(t-c)}}{\left(s-a_{v}\right)^{\mu+1} \Omega(s)}=\frac{e^{a_{\nu}(t-c)}}{\left(s-a_{v}\right)^{\mu+1}} \sum_{j=0}^{\infty} \Gamma_{j}\left(s-a_{v}\right)^{i},
$$

where $\Gamma_{j}$ is obtained by convolution of the sequences $\left\{\omega_{j}\right\}_{0}^{\infty}$ and $\left\{(t-c)^{i} / j !\right\}_{0}^{\infty}$ :

$$
\Gamma_{\mu}=\omega_{0} \frac{(t-c)^{\mu}}{\mu !}+\cdots+\omega_{\mu}
$$

This is a real polynomial of degree $\mu$ with leading coefficient $\omega_{0} / \mu$ !. Since

$$
R_{\nu}=\Gamma_{\mu} e^{a_{\nu}(t-c)},
$$

our theorem is proved.

6. The changes of sign of the derivatives of $G_{m, n}(t)$. In this section and the next we shall study the graphs of the functions $G_{m, n}(t)$ and $G_{m}(t)$. We shall show that they are "bell-shaped" (like $e^{-x^{2}}$ for example). By an exponential polynomial we shall mean a sum of the form 


$$
Q_{d}(t)=\sum_{k=1}^{n} e^{a_{k} t} P_{k}(t)
$$

where the $a_{k}$ are real and distinct and $P_{k}(t)$ is a polynomial of degree $m_{k}$. The degree of the exponential polynomial is defined to be

$$
d=-1+\sum_{k=1}^{n}\left(m_{k}+1\right) .
$$

Observe that $d$ is a unit less than the maximum possible number of terms in $Q_{d}(t)$ if each $P_{k}(t)$ is expanded in powers of $t$. If $Q_{d}(t)$ reduces to an ordinary polynomial then $d$ is its degree in the usual sense.

TheOREM 6a. If $G_{m, n}(t)$ is defined as in Theorem $1 \mathrm{~b}$ with $n-m \geqq 2$ and if

$$
\begin{gathered}
\sigma_{m, n}=b_{m, n}+\sum_{k=m+1}^{n} \frac{1}{a_{k}}, \\
d_{1}=-1+\sum_{k=m+1, a_{k}>0}^{n} 1, \quad d_{2}=-1+\sum_{k=m+1, a_{k}<0}^{n} 1,
\end{gathered}
$$

then $G_{m, n}(t)$ is an exponential polynomial in each of the intervals $\left(-\infty, \sigma_{m, n}\right)$, $\left(\sigma_{m, n}, \infty\right)$ of degrees $d_{1}$ and $d_{2}$, respectively.

Let us choose $\lambda>\mu=\max \left(\left|a_{m+1}\right|,\left|a_{m+2}\right|, \cdots,\left|a_{n}\right|\right)$. Integrating around the rectangular contour with vertices $s= \pm i T, s=\lambda \pm i T$ in the positive sense we obtain

$$
\frac{1}{2 \pi i} \int_{D} \frac{e^{s t}}{E_{m, n}(s)} d s=-Q_{d_{1}}(t),
$$

where $Q_{d_{1}}(t)$ is minus the sum of the residues of the integrand at its positive poles. Reference to Theorem 5 shows that this sum is an exponential polynomial whose degree $d_{1}$ is the sum of the orders of the positive poles less unity. If we now allow $T$ to become infinite we see by use of Corollary 1c and Theorem $2 \mathrm{~b}$ that .

$$
\begin{aligned}
G_{m, n}(t) & =Q_{d_{1}}(t)+I \\
I=\frac{1}{2 \pi i} \int_{\lambda-i \infty}^{\lambda+i \infty} \frac{e^{s t}}{E_{m, n}(s)} d s & =\frac{1}{2 \pi i} \int_{\lambda-i \infty}^{\lambda+i \infty} \frac{e^{s\left(t-\sigma_{m, n}\right)}}{\prod_{k=m+1}^{n}\left(1-\frac{s}{a_{k}}\right)} d s .
\end{aligned}
$$

We shall now show that $I=0$ when $t<\sigma_{m, n}$. Clearly $I$ is independent of $\lambda$ for $\lambda>\mu$. If $\lambda \geqq 1+\mu$ and $n \geqq m+2$, then

$$
\left|1-\frac{\lambda+i \tau}{a_{k}}\right|=\left(\left(1-\frac{\lambda}{a_{k}}\right)^{2}+\frac{\tau^{2}}{a_{k}^{2}}\right)^{1 / 2} \geqq \frac{\left(1+\tau^{2}\right)^{1 / 2}}{\left|a_{k}\right|},
$$




$$
\prod_{k=m+1}^{n}\left|1-\frac{s}{a_{k}}\right| \geqq \frac{\left[1+\tau^{2}\right]^{(n-m) / 2}}{\prod_{k=m+1}^{n}\left|a_{k}\right|} \geqq \frac{\left[1+\tau^{2}\right]^{(n-m) / 2}}{\mu^{n-m}}
$$

Hence

$$
|I| \leqq \frac{\mu^{n-m}}{2 \pi} e^{\lambda\left(t-\sigma_{m}, n\right)} \int_{-\infty}^{\infty} \frac{d \tau}{\left[1+\tau^{2}\right]^{(n-m) / 2}} .
$$

Since $\lambda$ may be taken arbitrarily large we see that $I$ must be zero when $t-\sigma_{m, n}$ is negative. That is,

$$
G_{m, n}(t)=Q_{d_{1}}(t), \quad-\infty<t<\sigma_{m, n} .
$$

This equation holds for $t=\sigma_{m, n}$ by continuity.

In a similar way we may establish that, for $\sigma_{m, n} \leqq t<\infty, G_{m, n}(t)$ is an exponential polynomial which is equal to the sum of the residues of $e^{s t} / E_{m, n}(s)$ at the negative poles and hence of degree $d_{2}$ as stated in the theorem.

CoRollaRy 6b. If no $a_{k}$ is positive $\left(\alpha_{2}=+\infty\right)$, then $G_{m, n}(t) \equiv 0$ for $t \leqq \sigma_{m, n}$; if no $a_{k}$ is negative $\left(\alpha_{1}=-\infty\right)$, then $G_{m, n}(t) \equiv 0$ for $t \geqq \sigma_{m, n}$.

COROLlaRY 6c. If $\alpha_{1}$, as defined in Theorem $1 \mathrm{~b}$, is a zero of order $\mu_{1}+1$ of $E_{m, n}(s)$, then

$$
\lim _{t \rightarrow+\infty} \frac{G_{m, n}(t)}{e^{\alpha_{1}} t \mu_{1}}=A_{1}>0
$$

if $\alpha_{2}$ is a zero of order $\mu_{2}+1$, then

$$
\lim _{t \rightarrow-\infty}(-1)^{\mu_{2}} \frac{G_{m, n}(t)}{e^{\alpha_{2} t} t^{\mu_{2}}}=A_{2}>0
$$

By reference to Theorem 5 we can obtain $A_{1}$ explicitly:

$$
A_{1}=\frac{\left(-\alpha_{1}\right)^{\mu_{1}+1}}{\mu_{1} !} e^{-\alpha_{1} c} \prod_{k=m+1, a_{k \neq \alpha_{1}}}^{n}\left(1-\frac{\alpha_{1}}{a_{k}}\right)^{-1} e^{-\alpha_{1} / a_{k}}, \quad \cdot c=b_{m, n}+\frac{\mu_{1}+1}{\alpha_{1}} .
$$

Since $\alpha_{1}$ is the largest of the negative $a_{k}$ and is itself negative it is evident by inspection that $A_{1}>0$. In a similar way $A_{2}$ may be exhibited explicitly and may be seen to be positive.

The following result is a familiar one, see [12, vol. 2 , p. 48]. Its proof is included here for completeness.

Lemma 6d. If $Q_{d}(t)$ is an exponential polynomial of degree d, it can have at most d real zeros.

It is to be understood, as is usual, that the number of zeros is the sum of 
the orders of the distinct zeros. We prove the result by induction. It is obvious if $d=0$. Assume it true for degree $d-1$. Now suppose that $Q_{d}(t)$, as defined in $\S 6$, had $d+1$ zeros. The same would be true of $e^{-a_{1} t} Q_{d}(t)$. By Rolle's theorem the derivative of this product would have at least $d$ zeros. But this derivative, being an exponential polynomial of degree $d-1$, cannot have $d$ zeros, by the assumption of the induction. The contradiction proves the lemma.

Definition 6e. A function $F(t)$ which is continuous for all $t$ has $r$ changes of sign if and only if there is a subdivision of the interval $(-\infty, \infty)$ into $r+1$ subintervals in each of which $F(t) \not \equiv 0$ and such that $F(t)$ has opposite signs in adjoining intervals.

Obvious modifications of the definition are needed if the interval in question is finite or semi-finite.

THEOREM 6f. If

$$
\begin{aligned}
& \text { 1. } E_{m, n}(s)=e^{b_{m, n},} \prod_{k=m+1}^{n}\left(1-\frac{s}{a_{k}}\right) e^{s / a_{k}}, \quad n-m \geqq 2, \\
& \text { 2. } G_{m, n}(t)=\frac{1}{2 \pi i} \int_{-i \infty}^{i \infty} \frac{e^{s t}}{E_{m, n}(s)} d s,
\end{aligned}
$$

then for $r=0,1, \cdots, n-m-2, G_{m, n}^{(r)}(t)$ has exactly $r$ changes of sign.

In Corollary $1 \mathrm{~d}$ we saw that $G_{m, n}(t) \in C^{n-m-2}$. By Corollaries $6 \mathrm{~b}$ and $6 \mathrm{c}$ it is clear that $G_{m, n}( \pm \infty)=0$. By Rolle's theorem $G_{m, n}^{\prime}(t)$ changes sign at least once. Again by use of the same two corollaries we see that $G_{m, n}^{\prime}( \pm \infty)=0$. The derivative can be computed explicitly from the known exponential polynomial expansions of $G_{m, n}(t)$. This calculation is facilitated by the fact that we are interested only in the leading term (dominant near $+\infty$ or $-\infty$ ). By Rolle's theorem $G_{m, n}^{\prime \prime}(t)$ changes sign at least twice. Proceeding in this way we show that for $r=0,1, \cdots, n-m-2, G_{m, n}^{(r)}(t)$ has at least $r$ changes of sign; we must show that it does not have more. If for any such $r$ it had more than $r$ changes of sign, then it would follow by Rolle's theorem that $G_{m, n}^{(n-m-2)}(t)$ would have more than $n-m-2$ changes of sign. We shall show this impossible.

Case 1. There are zeros of $E_{m, n}(s)$ on both sides of the origin $\left(-\infty<\alpha_{1}\right.$ $\left.<\alpha_{2}<\infty\right)$. Then

$$
\begin{array}{rlrl}
G_{m, n}^{(n-m-2)}(t) & =Q_{d_{1}}^{(n-m-2)}(t) & \left(-\infty<t \leqq \sigma_{m, n}\right) \\
& =Q_{d_{2}}^{(n-m-2)}(t) \quad\left(\sigma_{m, n} \leqq t<\infty\right)
\end{array}
$$

where $d_{1}, d_{2}, \sigma_{m, n}$ were defined in Theorem 6a. Clearly $d_{1} \geqq 0, d_{2} \geqq 0$. By Lemma $6 \mathrm{~d}, Q_{d_{1}}^{(n-m-2)}(t)$ has at most $d_{1}$ zeros in $-\infty<t<\infty$ and $a$ fortiori, at 
most $d_{1}$ changes of sign in $\left(-\infty, \sigma_{m, n}\right)$. In a similar way $Q_{d_{2}}^{(n-m-2)}(t)$ has at most $d_{2}$ changes of sign in $\left(\sigma_{m, n}, \infty\right)$. That is, $G_{m, n}^{(n-m-2)}(t)$ has at most $d_{1}+d_{2}=n-m-2$ changes of sign in $(-\infty, \infty)$. [Observe that the degree of an exponential polynomial of the form (1), $\$ 6$, with all $a_{k} \neq 0$ is unchanged by differentiation.]

Case 2. The zeros of $E_{m, n}(s)$ are all on one side of the origin $\left(\alpha_{1}=-\infty\right.$ or $\left.\alpha_{2}=+\infty\right)$. Suppose that $\alpha_{1}=-\infty$. The case $\alpha_{2}=+\infty$ is similar and may be omitted. Then $Q_{d_{2}}(t) \equiv 0$ and $d_{1}=n-m-1$. That is, $G_{m, n}^{(n-m-2)}(t)$ will have at most $n-m-1$ zeros in $-\infty<t \leqq \sigma_{m, n}$. At least one of these zeros is at $\sigma_{m, n}$ by continuity, but this one is not a change of sign. Hence $G_{m, n}^{(n-m-2)}(t)$ has at most $n-m-2$ changes of sign. As in Case 1 the same result holds for the derivatives of $G_{m, n}(t)$ of order $\leqq n-m-2$. In both cases it is important for the success of the argument that $G_{m, n}(t) \in C^{n-m-2}$. The proof is now complete.

7. Intersection properties of the $G_{m, n}(t)$. If in the function $G_{m, n}(t)$ we fix the subscript $m$ and allow $n$ to vary, keeping $n \geqq m+2$, we obtain a family of functions with bell-shaped graphs which have, as we shall show, the property that two distinct graphs cross each other at most twice. Since the constants $a_{k}$ appearing in the definition of $G_{m, n}(t)$ are not assumed to be arranged in any special order it will be evident that when $n$ is kept fixed and $m$ allowed to vary a new family of functions with the same intersection property is obtained.

Theorem 7. If $G_{m, n}(t)$ is defined as in Theorem $1 \mathrm{~b}$, if $p-m \geqq 2, q-m \geqq 2$, and if $p \neq q$, then the function

$$
\phi(t)=G_{m, p}(t)-G_{m, q}(t)
$$

has at most two changes of sign.

Assume for definiteness that $p>q$. By Corollary $1 \mathrm{~d}, \phi(t) \in C^{q-m-2}$. Now suppose that $\phi(t)$ had more than two changes of sign. Then by Rolle's theorem the function

$$
(1-D / a) \phi(t)=-(1 / a) e^{a t} D e^{-a t} \phi(t)
$$

would have at least three changes of sign. In making this calculation one must take into account the fact that $e^{-a t} \phi(t)$ vanishes either at $+\infty$ or at $-\infty$. Proceeding step by step in this way we see that the continuous function

$$
\begin{aligned}
\prod_{k=m+1}^{q-2}\left(1-\frac{D}{a_{k}}\right) \phi(t)= & \frac{1}{2 \pi i} \int_{-i \infty}^{i \infty} \frac{e^{s\left(t-\sigma_{m}, p\right)}}{\prod_{q-1}^{p}\left(1-\frac{s}{a_{k}}\right)} d s \\
& -\frac{1}{2 \pi i} \int_{-i \infty}^{i \infty} \frac{e^{s\left(t-\sigma_{m, q}\right)}}{\left(1-\frac{s}{a_{q-1}}\right)\left(1-\frac{s}{a_{q}}\right)} d s
\end{aligned}
$$


would have at least three changes of sign. We shall show this impossible.

Case 1. $a_{q} a_{q-1}>0$. Suppose for definiteness that $a_{q}<0, a_{q-1}<0$. Then the changes of sign in $\phi(t)$ must be in the interval $\left(\sigma_{m, q}, \infty\right)$. For, in this case the second of the integrals (2) is identically zero in $\left(-\infty, \sigma_{m, q}\right)$ by Corollary $6 \mathrm{~b}$, and the first never changes sign there by Corollary 6f. But $\phi(t) \in C^{p-m-2}$, at least, in the interval $\left(\sigma_{m, q}, \infty\right)\left(\phi(t) \in C^{\infty}\right.$ there if $\left.\sigma_{m, p}<\sigma_{m, q}\right)$. Hence we may apply the above argument two steps further to conclude that the function

$$
\prod_{k=m+1}^{q}\left(1-\frac{D}{a_{k}}\right) \phi(t)=\frac{1}{2 \pi i} \int_{-i \infty}^{i \infty} \frac{e^{s\left(t-\sigma_{m}, p\right)}}{\prod_{k=q+1}^{p}\left(1-\frac{s}{a_{k}}\right)} d s
$$

must have at least one change of sign, contrary to Theorem $6 f$. Observe that we have tacitly assumed here that $p \geqq q+2$. If $p=q+1$ then we must appeal to an extended form of Rolle's theorem which allows the derivative to be only piecewise continuous providing that at a point of discontinuity the derivative is by convention declared to assume all values between the left and right hand limits. If $a_{q}$ and $a_{q-1}$ were both positive the argument could be altered in an obvious way.

Case 2. $a_{q} a_{q-1}<0$. First suppose $a_{q}>0, a_{q-1}<0$. In one of the intervals $\left(-\infty, \sigma_{m, q}\right),\left(\sigma_{m, q}, \infty\right), \phi(t)$ must have at least two changes of sign. Suppose it is in the latter. Then the function

$$
\prod_{k=m+1}^{q-1}\left(1-\frac{D}{a_{q-1}}\right) \phi(t)=\frac{1}{2 \pi i} \int_{-i \infty}^{i \infty} \frac{e^{s\left(t-\sigma_{m}, p\right)}}{\prod_{k=q}^{p}\left(1-\frac{s}{a_{k}}\right)} d s
$$

must have at least one change of sign in $\left(\sigma_{m q}, \infty\right)$. This again contradicts Theorem $6 \mathrm{f}$. The other cases are handled in a similar way, so that the theorem is established.

8. The $G_{m, n}(t)$ as frequency functions. A function $f(t)$ defined for $-\infty<t<\infty$ is said to be a frequency function (see [6, pp. 166-178] for example) if $f(t) \geqq 0$ and $\int_{-\infty}^{\infty} f(t) d t=1$. The mean value and variance are defined to be, respectively,

$$
\mu=\int_{-\infty}^{\infty} t f(t) d t, \quad \nu=\int_{-\infty}^{\infty}(t-\mu)^{2} f(t) d t
$$

It is well known (see [6; pp. 188-192]) that if $f_{1}(t)$ and $f_{2}(t)$ are frequency functions with mean values $\mu_{1}, \mu_{2}$ and variances $\nu_{1}, \nu_{2}$, respectively, then the convolution of $f_{1}(t)$ with $f_{2}(t)$,

$$
f_{1} * f_{2}(t)=\int_{-\infty}^{\infty} f_{1}(t-u) f_{2}(u) d u
$$


is a frequency function with mean value $\mu_{q}+\mu_{2}$ and with variance $\nu_{1}+\nu_{2}$. By use of this result we can now prove the following theorem.

THEOREM 8. If

$$
G_{m, n}(t)=\frac{1}{2 \pi i} \int_{-\infty}^{\infty} \frac{e^{s t} d s}{e^{b_{m, n} s} \prod_{k=m+1}^{n}\left(1-\frac{s}{a_{k}}\right) e^{s / a_{k}}},
$$

then $G_{m, n}(t)$ is a frequency function with mean value $b_{m, n}$ and with variance $\sum_{k=m+1}^{n} a_{k}^{-2}$.

Since, by Theorem $1 \mathrm{~b}$, we have

$$
G_{m, n}(t)=g_{m+1} * g_{m+2} * \cdots * g_{n}\left(t-b_{m, n}\right),
$$

it will, by virtue of the result quoted above, be sufficient to establish that $g_{k}(t) \geqq 0$ and that

$$
\int_{-\infty}^{\infty} g_{k}(t) d t=1, \quad \int_{-\infty}^{\infty} t_{k}(t) d t=0, \quad \int_{-\infty}^{\infty} t^{2} g_{k}(t) d t=1 / a_{k}^{2} .
$$

For, after the convolutions are performed, the change of variable $t-b_{m, n}=u$ will give the desired result. But using the definition of $g_{k}(t)$ we have by simple calculations, say for $a_{k}>0$,

$$
\begin{aligned}
& \int_{-\infty}^{\infty} g_{k}(t) d \imath=a_{k} \int_{-\infty}^{1 / a_{k}} e^{a_{k} t-1} d t=1, \\
& \int_{-\infty}^{\infty} t g_{k}(t) d t=a_{k} \int_{-\infty}^{1 / a_{k}} t e^{u_{h} t-1} d t=0, \\
& \int_{-\infty}^{\infty} t^{2} g_{k}(t) d \iota=a_{k} \int_{-\infty}^{1 / a_{k}} t^{2} e^{a_{l} t-1} d t=1 / a_{k}^{2} .
\end{aligned}
$$

Since changing $a_{k}$ to $-a_{k}$ replaces $g_{k}(t)$ by $g_{k}(-t)$, the theorem is seen to hold in all cases.

9. Asymptotic estimates for $G_{m}(t)$. In this section we shall employ Theorem 5 to obtain the dominant term in asymptotic expansions for $G_{m}(t)$ as $t \rightarrow+\infty$, and as $t \rightarrow-\infty$. These results are fundamental in our theory and will be used extensively. We need them here in order to extend the results of the preceeding sections to $G_{m}(t)$.

Definition 9a. Let $E_{m}(s)$ be defined as in Theorem 3a. We set

$$
\begin{array}{ll}
\alpha_{1}(m)=\max _{a_{k}<0}\left[a_{k},-\infty\right], & k=m+1, m+2, \cdots ; \\
\alpha_{2}(m)=\min _{a_{k}>0}\left[a_{k},+\infty\right], & k=m+1, m+2, \cdots ;
\end{array}
$$




$$
\begin{aligned}
& \mu_{1}(m)+1=\text { multiplicity of } \alpha_{1}(m) \text { as a zero of } E_{m}(s) ; \\
& \mu_{2}(m)+1=\text { multiplicity of } \alpha_{2}(m) \text { as a zero of } E_{m}(s) .
\end{aligned}
$$

Note that $\alpha_{1}(0)$ and $\alpha_{2}(0)$ coincide with $\alpha_{1}$ and $\alpha_{2}$ as previously defined.

Theorem 9b. Let $G_{m}(t)$ be defined as in Theorem 3a, and $\alpha_{1}(m), \alpha_{2}(m)$, $\mu_{1}(m)$, and $\mu_{2}(m)$ as in Definition 9a. Then

A. $\alpha_{1}(m) \neq-\infty$ implies

$$
\left(\frac{d}{d t}\right)^{r} G_{m}(t)=\left(\frac{d}{d t}\right)^{r}\left[p_{m}(t) e^{\alpha_{1}(m) t}\right]+O\left[e^{\alpha_{1}(m) t-\epsilon t}\right]
$$

as $t \rightarrow+\infty, r=0,1,2, \cdots$, where $\epsilon$ is a positive constant and $p_{m}(t)$ is a real polynomial of degree $\mu_{1}(m)$ with the coefficient of $t^{\mu_{1}(m)}$ positive;

B. $\alpha_{1}(m)=-\infty$ implies

$$
\left(\frac{d}{d t}\right)^{r} G_{m}(t)=O\left(e^{-k t}\right)
$$

as $t \rightarrow+\infty, r=0,1,2, \cdots$, for every $k$;

C. $\alpha_{2}(m) \neq+\infty$ implies

$$
\left(\frac{d}{d t}\right)^{r} G_{m}(t)=\left(\frac{d}{d t}\right)^{r}\left[q_{m}(t) \epsilon^{\alpha_{2}(m) t}\right]+O\left[e^{\alpha_{2}(m) t+\epsilon t}\right]
$$

as $t \rightarrow-\infty, r=0,1,2, \cdots$, where $\epsilon$ is a positive constant and $q_{m}(t)$ is a real polynomial of degree $\mu_{2}(m)$ with the coefficient of $(-t)^{\mu_{2}(m)}$ positive;

D. $\alpha_{2}(m)=+\infty$ implies

$$
\left(\frac{d}{d t}\right)^{r} G_{m}(t)=O\left(e^{k t}\right)
$$

as $t \rightarrow-\infty, r=0,1,2, \cdots$, for every $k$.

Let us demonstrate conclusion A. We choose any fixed $\lambda$ such that

$$
\alpha_{1}(m)>\lambda>\max _{a_{k}<0, a_{k} \neq \alpha_{1}(m)}\left[a_{k}\right] . \quad k=m+1, m+2, \cdots .
$$

Integrating in the positive direction about the rectangular contour $D$ with vertices at $\pm i T, \lambda \pm i T$, we find using Theorem $5(n=+\infty)$ that

$$
\frac{1}{2 \pi i} \int_{D} \frac{e^{s t}}{E_{m}(s)} d s=p_{m}(t) e^{\alpha_{1}(m) t},
$$

where $p_{m}(t)$ is a real polynomial of degree $\mu_{1}(m)$. The coefficient of $t^{\mu_{1}(m)}$ in $p_{m}(t)$ is positive since $p$ was positive in Theorem 5 . Using Theorems $2 b$ and $3 a$ and letting $T$ increase without limit we obtain 


$$
G_{m}(t)=p_{m}(t) e^{\alpha_{1}(m) t}+\frac{1}{2 \pi i} \int_{\lambda-i \infty}^{\lambda+i \infty} \frac{e^{s t}}{E_{m}(s)} d s .
$$

Differentiating $r$ times, this becomes

$$
\left(\frac{d}{d t}\right)^{r} G_{m}(t)=\left(\frac{d}{d t}\right)^{r}\left[p_{m}(t) e^{\alpha_{1}(m) t}\right]+\frac{1}{2 \pi i} \int_{\lambda-i \infty}^{\lambda+i \infty} \frac{s^{r} e^{s t}}{E_{m}(s)} d s
$$

By Theorem $2 \mathrm{~b}$ there is a constant $c$ such that

$$
\left|\frac{1}{E_{m}(\lambda+i \tau)}\right| \leqq \frac{c}{1+|\tau|^{+2}} \quad(-\infty<\tau<\infty) .
$$

Hence

$$
\begin{aligned}
\left|\frac{1}{2 \pi i} \int_{\lambda-i \infty}^{\lambda+i \infty} \frac{s^{r} e^{s t}}{E_{m}(s)} d s\right| & \leqq \frac{c}{2 \pi} e^{+\lambda t} \int_{-\infty}^{\infty} \frac{\left[\lambda^{2}+\tau^{2}\right] r / 2}{|\tau|^{r+2}+1} d \tau \quad(t>0) \\
& =O\left[e^{\alpha_{1}(m) t} e^{-\tau t}\right] \quad \text { as } t \rightarrow+\infty,
\end{aligned}
$$

where $+\alpha_{1}(m)-\lambda=\epsilon$. This proves conclusion A.

If $\alpha_{1}(m)=-\infty$, and if we put $\lambda=-k$, we may by an identical procedure demonstrate conclusion $B$. Here of course the residue is identically zero. Similarly we may dispose of conclusions $C$ and $D$. $t \geqq \sigma_{m}$,

CoROllary 9c. If $\alpha_{1}(m)=-\infty$ and $\sum_{k=1}^{\infty} 1 / a_{k}<\infty$, then $G_{m}(t)=0$ for

$$
\sigma_{m}=b_{m}+\sum_{k=m+1}^{\infty} 1 / a_{k}
$$

if $\alpha_{2}(m)=+\infty$ and $\sum_{k=1}^{\infty} 1 / a_{k}>-\infty$, then $G_{m}(t)=0$ for $t \leqq \sigma_{m}$.

Suppose that $\alpha_{1}(m)=-\infty$. Let $G_{m, n}(t)$ be defined as in Theorem 1b with $b_{m, n}=b_{m}$. By Corollary $6 \mathrm{~b}, G_{m, n}(t)=0, t \geqq b_{m}+\sum_{k=m+1}^{\infty} 1 / a_{k}=\sigma_{m}$. By Theorem $4 \mathrm{a}$

$$
\lim _{n \rightarrow \infty} G_{m, n}(t)=G_{m}(t), \quad-\infty<t<\infty .
$$

Hence $G_{m}(t)=0, t \geqq \sigma_{m}$. The case $\alpha_{2}(m)=+\infty$ is entirely similar.

10. The changes of sign of the derivatives of $G_{m}(t)$. Using the results of $\$ 4$ we may extend the properties obtained for $G_{m, n}(t)$ in $\$ 6$ to the limit function $G_{m}(t)$.

TheOREM 10. If $G_{m}(t)$ is defined as in Theorem 3a, then $G_{m}^{(r)}(t)$ has precisely $r$ changes of sign $(-\infty<t<\infty)$.

By Corollary $3 \mathrm{~b}, G_{m}(t) \in C^{\infty}$. Using the asymptotic estimates for $G_{m}^{(r)}(t)$ as 
$t \rightarrow \pm \infty, r=0,1,2, \cdots$, derived in $\$ 9$, and Rolle's theorem we see that $G_{m}^{(\tau)}(t)$ must have at least $r$ changes of sign. On the other hand by $\$ 4$

$$
G_{m}^{(r)}(t)=\lim _{n \rightarrow \infty} G_{m, n}^{(r)}(t) \quad(-\infty<t<\infty),
$$

if we suppose that $\lim _{n \rightarrow \infty} b_{m, n}=b_{m}$. By Theorem 6 f, $G_{m, n}^{(r)}(t)$ has for $n \geqq m+r+2$ exactly $r$ changes of sign. As the limit of functions, each with no more than $r$ changes of sign, $G_{m}(t)$ must have $r$ or fewer changes of sign; hence it has precisely $r$.

11. Intersection properties of $G_{m}(t)$. Theorem 7 may also be extended to the infinite case.

Theorem 11. If $G_{m}(t)$ is defined as in Theorem 3a then for $p \neq q, G_{p}(t)$ $-G_{q}(t)$ has at most two changes of sign $(-\infty<t<\infty)$.

This is proved exactly as Theorem 10 was proved. We have

$$
G_{p}(t)-G_{q}(t)=\lim _{n \rightarrow \infty}\left[G_{p, n}(t)-G_{q, n}(t)\right]
$$

provided $\lim _{n \rightarrow \infty} b_{m, n}=b_{m}$. By Theorem 7 the functions $G_{p, n}(t)-G_{q, n}(t)$ have at most two changes of sign, which implies that $G_{p}(t)-G_{q}(t)$ has at most two changes of sign.

12. The $G_{m}(t)$ as frequency functions.

THEOREM 12. If $G_{m}(t)$ is defined as in Theorem 3a then $G_{m}(t)$ is a frequency function with mean value $b_{m}$ and variance $S_{m}=\sum_{k=m+1}^{\infty} a_{k}^{-2}$.

By Corollary $4 \mathrm{~b}, G_{m}(t) \geqq 0(-\infty<t<\infty)$. By Theorem 3a we have

$$
\int_{-\infty}^{\infty} e^{-s\left(t-b_{m}\right)} G_{m}(t) d t=\frac{1}{\prod_{k=m+1}^{\infty}\left(1-\frac{s}{a_{k}}\right) e^{s / a_{k}}}
$$

the bilateral Laplace transform (1) converging absolutely for $\alpha_{1}(m)<s$ $<\alpha_{2}(m)$. Let us define

$$
\begin{aligned}
& R_{0}(s)=\int_{-\infty}^{\infty} e^{-s\left(t-b_{m}\right)} G_{m}(t) d t \\
& R_{1}(s)=\int_{-\infty}^{\infty} e^{-s\left(t-b_{m}\right)}\left[t-b_{m}\right] G_{m}(t) d t \\
& R_{2}(s)=\int_{-\infty}^{\infty} e^{-s\left(t-b_{m}\right)}\left[t-b_{m}\right]^{2} G_{m}(t) d t
\end{aligned}
$$

Using equation (1) we see that $R_{0}(0)=1$, so that $G_{m}(t)$ is indeed a frequency 
function. Computing the logarithmic derivative of equation (1) we obtain

$$
R_{1}(s) / R_{0}(s)=-\sum_{m+1}^{\infty} \frac{s}{a_{k}\left(a_{k}-s\right)} .
$$

If we set $s=0$ in equation (2) we find $R_{1}(0)=0$, or $G_{m}(t)$ has mean $t=b_{m}$. Differentiating equation (2) we obtain

$$
\left[R_{0}(s) R_{2}(s)-R_{1}(s)^{2}\right] / R_{0}(s)^{2}=\sum_{k=m+1}^{\infty} \frac{1}{\left(a_{k}-s\right)^{2}} .
$$

Putting $s=0$ we see that $R_{2}(0)=\sum_{m+1}^{\infty} a_{k}^{-2}$ or $G_{m}(t)$ has variance $\sum_{m+1}^{\infty} a_{k}^{-2}$.

13. Some further properties of $G_{m}(t)$.

Theorem 13a. If $G_{m}(t)$ is defined as in Theorem 3a, if $\zeta_{m}$ is a point associated, in the sense of Definition 6e, with the one change of sign of $G_{m}^{\prime}(t)$, and if $\zeta_{m}^{(1)}$ and $\zeta_{m}^{(2)}, \zeta_{m}^{(1)}<\zeta_{m}^{(2)}$, are two points associated respectively with the two changes of sign of $G_{m}^{\prime \prime}(t)$, then

A. $G_{m}^{\prime}(t)$ is not identically zero in any closed interval interval containing $\zeta_{m}$; or $\zeta_{m}^{(2)}$.

B. $G_{m}^{\prime \prime}(t)$ is not identically zero in any closed interval containing either $\zeta_{m}^{(1)}$

Our theorem is equivalent to the statement that the points $\zeta_{m}, \zeta_{m}^{(1)}$ and $\zeta_{m}^{(2)}$ are uniquely determined. A consideration of the graphs of $G_{m}^{\prime}(t), G_{m}^{\prime \prime}(t)$, and $G_{m}^{\prime \prime \prime}(t)$ will show that should $G_{m}^{\prime}(t)$ be identically zero in a closed interval containing $\zeta_{m}$, then $G_{m}^{\prime \prime \prime}(t)$ would have five changes of sign, whereas we know that it has only three. This establishes conclusion A.

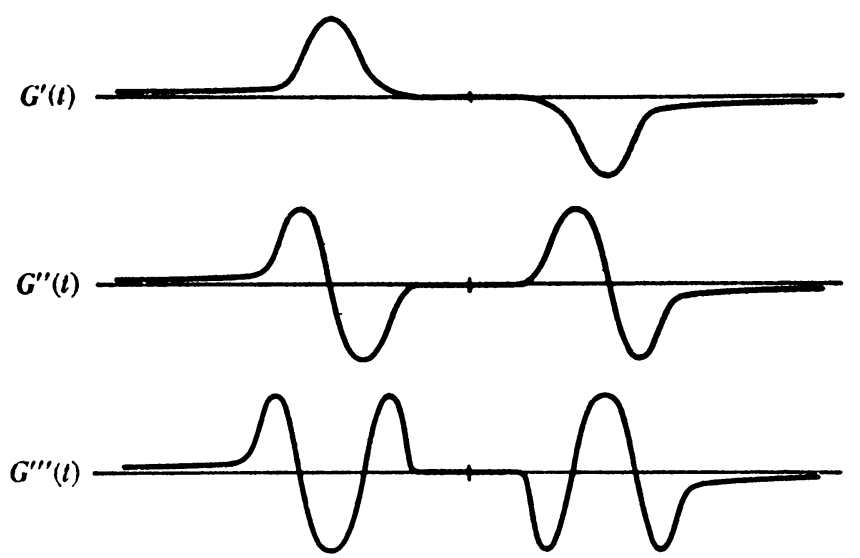

Conclusion B follows from a similar argument, which we leave to the reader. 
THEOREM 13b. If $G_{m}(t)$ is defined as in Theorem 3a, and if $\zeta_{m}, \zeta_{m}^{(1)}$ and $\zeta_{m}^{(2)}$ are defined as in Theorem 13a, then
A. $\lim _{m \rightarrow \infty} G_{m}(t)=0$
$(0<|t|<\infty)$
B. $\left|\zeta_{m}-b_{m}\right| \leqq 8\left[\sum_{k=m+1}^{\infty} \frac{1}{a_{k}^{2}}\right]^{1 / 2}$;
C. $\left|\zeta_{m}^{(i)}-b_{m}\right| \leqq 19\left[\sum_{k=m+1}^{\infty} \frac{1}{a_{k}^{2}}\right]^{1 / 2}$,
$i=1,2$.

Note that in the presence of our assumptions conclusions B and C imply that $\zeta_{m}, \zeta_{m}^{(1)}$ and $\zeta_{m}^{(2)}$ tend to zero with $1 / m$. Set $S_{m}=\sum_{k=m+1}^{\infty} 1 / a_{k}, \epsilon=2 S_{m}^{1 / 2}$. Then by Theorem 12

$$
\begin{aligned}
\int_{-\epsilon}^{\epsilon} G_{m}\left(t+b_{m}\right) d t & =1-\int_{\epsilon}^{\infty} G_{m}\left(t+b_{m}\right) d t-\int_{-\infty}^{-\epsilon} G_{m}\left(t+b_{m}\right) d t \\
& \geqq 1-\frac{1}{\epsilon^{2}} \int_{\epsilon}^{\infty} t^{2} G_{m}\left(t+b_{m}\right) d t-\frac{1}{\epsilon^{2}} \int_{-\infty}^{-\epsilon} t^{2} G_{m}\left(t+b_{m}\right) d t \\
& \geqq 1-\frac{1}{\epsilon^{2}} \int_{-\infty}^{\infty} t^{2} G_{m}\left(t+b_{m}\right) d t=1-\frac{S_{m}}{\epsilon^{2}}
\end{aligned}
$$

From this it follows that

$$
\max _{|l| \leqq \epsilon} G_{m}\left(t+b_{m}\right) \geqq \frac{3}{8 \epsilon} .
$$

We wish to prove that $\left|\zeta_{m}-b_{m}\right| \leqq 4 \epsilon$. Suppose the contrary. Then, since $G_{m}(t)$ has a unique maximum at $\zeta_{m}$, it is monotonic in the interval from $b_{m}$ to $\zeta_{m}$, and hence bigger near $\zeta_{m}$ than in the interval $\left|b_{m}-t\right| \leqq \epsilon$. That is, $G_{m}(t) \geqq 3 / 8 \epsilon$ throughout an interval of length $3 \epsilon$ with $\zeta_{m}$ as one end point. Hence

$$
1=\int_{-\infty}^{\infty} G_{m}(t) d t=\int_{-\infty}^{\infty} G_{m}\left(t+b_{m}\right) d t \geqq(3 \epsilon)\left(\frac{8}{3 \epsilon}\right)=\frac{9}{8} .
$$

This is a contradiction. Hence conclusion B is established.

Let $t_{0}$ be an arbitrary number different from zero and choose $m$ so large that $\left|b_{m}\right|<\left|t_{0}\right| / 2$. Then

$$
\begin{aligned}
\left|\int_{t_{0}}^{t_{0} / 2} G_{m}(t) d t\right| & \leqq \int_{|t| \geqq\left|t_{0}\right| / 2} G_{m}(t) d t \leqq\left(\frac{\left|t_{0}\right|}{2}-\left|b_{m}\right|\right)^{-2} \int_{-\infty}^{\infty}\left(t-b_{m}\right)^{2} G_{m}(t) d t \\
& \leqq\left(\frac{\left|t_{0}\right|}{2}-\left|b_{m}\right|\right)^{-2} S_{m} .
\end{aligned}
$$


Hence

$$
\lim _{m \rightarrow \infty} \int_{t_{0}}^{t_{0} / 2} G_{m}(t) d t=0 .
$$

But if $m$ is so large that $\left|\zeta_{m}\right|<\left|t_{0}\right| / 2$, then $G_{m}(t)$ is monotonic over the range of this integral and takes its smallest value at $t_{0}$. That is,

$$
G_{m}\left(t_{0}\right) \cdot\left|t_{0}\right| / 2 \leqq\left|\int_{t_{0}}^{t_{0} / 2} G_{m}(t) d t\right|
$$

From this inequality and equation (2) conclusion $\mathrm{A}$ is immediate.

By Rolle's theorem we must have $\zeta_{m}^{(1)}<\zeta_{m}<\zeta_{m}^{(2)}$. Further $G_{m}(t)$ is concave downward between $\zeta_{m}^{(1)}$ and $\zeta_{m}^{(2)}$. Consequently $G_{m}(t)$ lies above the line segment connection $\left(\zeta_{m}^{(1)}, 0\right)$ and $\left(\zeta_{m}, G\left(\zeta_{m}\right)\right)$ for $\zeta_{m}^{(1)} \leqq t \leqq \zeta_{m}$, and above the segment connecting $\left(\zeta_{m}, G\left(\zeta_{m}\right)\right)$ and $\left(\zeta_{m}^{(2)}, 0\right)$ for $\zeta_{m} \leqq t \leqq \zeta_{m}^{(2)}$.

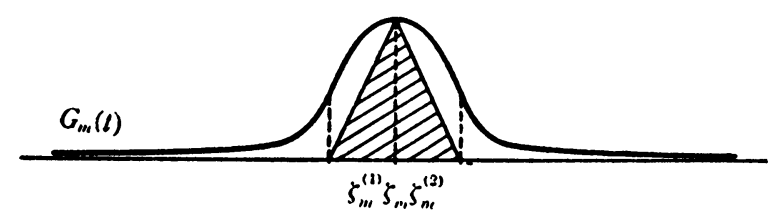

It follows that

$$
1=\int_{-\infty}^{\infty} G_{m}(t) d t \geqq\left[\zeta_{m}^{(2)}-\zeta_{m}^{(1)}\right] G_{m}\left(\zeta_{m}\right) / 2
$$

By equation (1)

$$
G_{m}\left(\zeta_{m}\right) \geqq \frac{3}{16 S_{m}^{1 / 2}}
$$

so that

$$
1 \geqq \frac{3}{32 S_{m}^{1 / 2}}\left[\zeta_{m}^{(2)}-\zeta_{m}^{(1)}\right]
$$

that is,

$$
\left[\zeta_{m}^{(2)}-\zeta_{m}^{(1)}\right] \leqq 11 S_{m}^{1 / 2}
$$

Using conclusion B and the fact that $\zeta_{m}$ lies between $\zeta_{m}^{(1)}$ and $\zeta_{m}^{(2)}$, we obtain conclusion $\mathrm{C}$.

14. Division into cases. For the remainder of this paper we shall consider three classes of kernels $G(t)$, for each of which separate treatment is required. 
Definition 14. $G(t)$ is said to belong to class I if $\alpha_{1} \neq-\infty, \alpha_{2} \neq+\infty$. $G(t)$ is said to belong to class II if $\alpha_{1}=-\infty, \sum_{1}^{\infty} 1 / a_{k}=+\infty . G(t)$ is said to belong to class III if $\alpha_{1}=-\infty, \sum_{1}^{\infty} 1 / a_{k}<\infty$.

It is evident that in every case either $G(t)$ or $G(-t)$ belongs to one of these three classes. The convolution transforms,

$$
f(x)=\int_{-\infty}^{\infty} G(x-t) d \alpha(t)
$$

constructed from class I kernels resemble the Stieltjes transform in their behavior. Those constructed from class II kernels resemble the Laplace transform. Transforms constructed from class III kernels are in some respects degenerate. There are no familiar examples for this class.

15. Convergence properties of class I kernels.

LemmA 15a. If $G(t)$ is a class I kernel, then for any fixed $x_{0}$ and any $x(-\infty<x<\infty)$ the integral

$$
\int_{-\infty}^{\infty}\left|\frac{d}{d t} \frac{G(x-t)}{G\left(x_{0}-t\right)}\right| d t
$$

converges, uniformly for $x$ in any finite interval $\left(x_{1} \leqq x \leqq x_{2}\right)$.

From Theorem 9b it follows that $G(t) \rightarrow 0$ as $t \rightarrow \pm \infty$, but that $G(t)$ is not identically zero in any neighborhood of $+\infty$ or $-\infty$. Since $G^{\prime}(t)$ has only one change of sign $G(t)$ must never vanish. Thus if $I(t)$ is the integrand (1), then

$$
I(t)=\left|\frac{-G\left(x_{0}-t\right) G^{\prime}(x-t)+G^{\prime}\left(x_{0}-t\right) G(x-t)}{G\left(x_{0}-t\right)^{2}}\right|,
$$

and $I(t)$ is continuous $(-\infty<t<\infty)$. By Theorem $9 \mathrm{~b}$

$$
\begin{aligned}
G\left(x_{0}-t\right) & =q\left(x_{0}-t\right) e^{\alpha_{2}\left(x_{0}-t\right)}+O\left[e^{\left(\alpha_{2}+t\right)\left(x_{0}-t\right)}\right], \\
G^{\prime}\left(x_{0}-t\right) & =\left[q\left(x_{0}-t\right) e^{\alpha_{2}\left(x_{0}-t\right)}\right]^{\prime}+O\left[e^{\left(\alpha_{2}+\epsilon\right)\left(x_{0}-t\right)}\right], \\
G(x-t) & =q(x-t) e^{\alpha_{2}(x-t)}+O\left[e^{\left(\alpha_{2}+t\right)(x-t)}\right], \\
G^{\prime}(x-t) & =\left[q(x-t) e^{\alpha_{2}(x-t)}\right]^{\prime}+O\left[e^{\left(\alpha_{2}+\epsilon\right)(x-t)}\right],
\end{aligned}
$$

as $t \rightarrow+\infty$, where $q(t)$ is a real polynomial of degree $\mu_{2}$. If equations (3) are inserted in (2) we find that

$$
\begin{aligned}
I(t) & =O\left(1 / t^{2}\right) & \left(\text { as } t \rightarrow+\infty, \text { if } \mu_{2}>0\right), \\
& =O\left(e^{-t t}\right) & \left(\text { as } t \rightarrow+\infty, \epsilon>0, \text { if } \mu_{2}=0\right),
\end{aligned}
$$

these estimates holding uniformly for $x$ in any finite interval. Similarly we may show that 


$$
\begin{aligned}
I(t) & =O\left(1 / t^{2}\right) & \left(\text { as } t \rightarrow-\infty, \text { if } \mu_{1}>0\right), \\
& =O\left(e^{\epsilon t}\right) & \left(\text { as } t \rightarrow-\infty, \epsilon>0, \text { if } \mu_{1}=0\right),
\end{aligned}
$$

these estimates holding uniformly for $x$ in any finite interval. Our lemma now follows.

THEOREM 15b. If

1. $G(t) \in$ class I,

2. $\int_{-\infty}^{\infty} G\left(x_{0}-t\right) d \alpha(t)$ converges, then

$$
\int_{-\infty}^{\infty} G(x-t) d \alpha(t)
$$

converges for all $x(-\infty<x<\infty)$, and converges uniformly for $x$ in any finite interval $\left(-\infty<x_{1} \leqq x<x_{2}<+\infty\right)$.

Let us set

$$
L(t)=\int_{0}^{t} G\left(x_{0}-u\right) d \alpha(u) .
$$

By assumption $2, L(t)$ is bounded and $L(+\infty)$ and $L(-\infty)$ exist. Since $G(t)$ never vanishes we have

$$
\begin{aligned}
\int_{T_{1}}^{T_{2}} G(x-t) d \alpha(t) & =\int_{T_{1}}^{T_{2}} \frac{G(x-t)}{G\left(x_{0}-t\right)} d L(t) \\
& =\left[\frac{G(x-t)}{G\left(x_{0}-t\right)} L(t)\right]_{T_{1}}^{T_{2}}-\int_{T_{1}}^{T_{2}}\left[\frac{G(x-t)}{G\left(x_{0}-t\right)}\right]^{\prime} L(t) d t .
\end{aligned}
$$

Using Theorem $9 \mathrm{~b}$ and the existence of $L(+\infty)$ and $L(-\infty)$, it is easily seen that

$$
\lim _{T_{1} \rightarrow-\infty, T_{2} \rightarrow+\infty}\left[\frac{G(x-t)}{G\left(x_{0}-t\right)} L(t)\right]_{T_{1}}^{T_{2}}
$$

exists uniformly for $\left(x_{1} \leqq x \leqq x_{2}\right)$. From Lemma 15a and the boundedness of $L(t)$

$$
\lim _{T_{1} \rightarrow-\infty, T_{2} \rightarrow+\infty} \int_{T_{1}}^{T_{2}}\left[\frac{G(x-t)}{G\left(x_{0}-t\right)}\right]^{\prime} L(t) d t
$$

exists uniformly for $\left(x_{1} \leqq x \leqq x_{2}\right)$. Thus

$$
\lim _{T_{1} \rightarrow-\infty, T_{2} \rightarrow+\infty} \int_{T_{1}}^{T_{2}} G(x-t) d \alpha(t)
$$

exists uniformly for $x$ in any finite interval, as desired 
THEOREM 15c. If

1. $G(t) \in$ class $\mathrm{I}$,

2. $\int_{-\infty}^{\infty} G\left(x_{0}-t\right) \phi(t) d t$ converges, then

$$
\int_{-\infty}^{\infty} G(x-t) \phi(t) d t
$$

converges for all $x(-\infty<x<\infty)$, and converges uniformly for $x$ in any finite interval $\left(-\infty<x_{1} \leqq x \leqq x_{2}<+\infty\right)$.

If we define

$$
\Phi(t)=\int_{0}^{t} \phi(u) d u
$$

then

$$
\int_{-\infty}^{\infty} G(x-t) \phi(t) d t=\int_{-\infty}^{\infty} G(x-t) d \Phi(t),
$$

and the present theorem follows from Theorem $15 \mathrm{~b}$.

16. Order properties of Riemann-Stieltjes integrals. The following theorem has been proved in many special cases, for example, Widder $[17$, pp. 3839 and 329-330], by exactly the method used here.

THEOREM 16. If

1. $\phi(t) \in C^{\prime}(0 \leqq t<\infty)$,

2. $\phi^{\prime}(t)$ is ultimately of constant sign as $t \rightarrow+\infty$,

3. $\int_{0}^{\infty} \phi(t) d \alpha(t)$ converges, then

A. if $\phi(t)$ approaches zero as $t \rightarrow \infty$ we have

$$
\alpha(t)=o[1 / \phi(t)]
$$$$
\text { as } t \rightarrow \infty \text {; }
$$

B. if $\phi(t)$ does not approach zero as $t \rightarrow \infty$ we have

$$
[\alpha(\infty)-\alpha(t)]=o[1 / \phi(t)]
$$$$
\text { as } t \rightarrow+\infty \text {. }
$$

Let us first consider conclusion A. We set

$$
L(t)=\int_{0}^{t} \phi(u) d \alpha(u)
$$

By assumption $3, L(\infty)$ exists. If $\phi(t)$ is identically zero in any neighborhood of $+\infty$ there is nothing to prove, so that we may suppose this is not the case. Consequently given $\epsilon>0$ we may choose $T$ so large that

$$
\phi(t) \neq 0
$$$$
(T \leqq t<\infty),
$$ 


$$
\begin{array}{lr}
\phi^{\prime}(t) \text { is of constant sign } & (T \leqq t<\infty), \\
\left|L(t)-L\left(t^{\prime}\right)\right|<\epsilon . & \left(T \leqq t, t^{\prime}<\infty\right) .
\end{array}
$$

For $t \geqq T$ we have

$$
\alpha(t)-\alpha(T)=\int_{T}^{t} \frac{1}{\phi(u)} d L(u),
$$

which after integration by parts becomes

$$
\alpha(t)=\alpha(T)+\frac{L(t)-L(T)}{\phi(T)}+\int_{T}^{t} \frac{\phi^{\prime}(u)}{\phi(u)^{2}}[L(u)-L(t)] d u .
$$

Now

$$
\begin{aligned}
\left|\int_{T}^{t} \frac{\phi^{\prime}(u)}{\phi(u)^{2}}[L(u)-L(t)] d u\right| & \leqq \max _{u, t \geqq T}|L(u)-L(t)| \int_{T}^{t}\left|\frac{\phi^{\prime}(u)}{\phi(u)^{2}}\right| d u \\
& <\epsilon\left|-\frac{1}{\phi(T)}+\frac{1}{\phi(t)}\right| \leqq \frac{\epsilon}{|\phi(t)|} .
\end{aligned}
$$

In case A, $\phi(t) \rightarrow 0$ as $t \rightarrow \infty$; hence for $t \geqq T$

$$
\begin{gathered}
|\alpha(t)|<\left|\frac{L(t)-L(T)}{\phi(T)}\right|+\left|\int_{T}^{t} \frac{\phi^{\prime}(u)}{\phi(u)^{2}}[L(u)-L(t)] d u\right|+|\alpha(T)| \\
<\epsilon\left|\frac{1}{\phi(t)}\right|+|\alpha(T)|+\epsilon\left|\frac{1}{\phi(T)}\right| \\
\underset{\limsup _{t \rightarrow \infty}|\alpha(t) \phi(t)| \leqq \epsilon .}{ }
\end{gathered}
$$

Since $\epsilon$ is arbitrary our result follows.

We now turn to $B$. In this case $\phi(t)$ is bounded away from zero for $t$ sufficiently large, so that there exist constants $T_{0}$ and $A, A>0$, such that

$$
|1 / \phi(t) \leqq A|
$$

Given $\epsilon>0$, we choose $T, T>T_{0}$, so large that for $(t \geqq T)$

$$
\begin{array}{lr}
\phi^{\prime}(t) \text { is of constant sign } & (T \leqq t<\infty), \\
\left|L(t)-L\left(t^{\prime}\right)\right| \leqq \frac{\epsilon}{3 A+2} & \left(T \leqq t, t^{\prime}<\infty\right) .
\end{array}
$$

When $t$ and $t^{\prime}$ are greater than $T$, we have

$$
\alpha(t)-\alpha\left(t^{\prime}\right)=\frac{L(t)-L\left(t^{\prime}\right)}{\phi\left(t^{\prime}\right)}+\int_{t^{\prime}}^{t} \frac{\phi^{\prime}(u)}{\phi(u)^{2}}[L(u)-L(t)] d u .
$$


From this we obtain, arguing as before,

$$
\left|\alpha(t)-\alpha\left(t^{\prime}\right)\right| \leqq 3 \epsilon A /(3 A+2)<\epsilon .
$$

Since $\epsilon$ is arbitrary this implies that $\alpha(\infty)$ exists. If, in equation (1), $t$ is allowed to increase without limit, we obtain

$$
\alpha(\infty)-\alpha\left(t^{\prime}\right)=\frac{L(\infty)-L\left(t^{\prime}\right)}{\phi\left(t^{\prime}\right)}+\int_{t^{\prime}}^{\infty} \frac{\phi^{\prime}(u)}{\phi(u)^{2}}[L(u)-L(\infty)] d u .
$$

There are two possibilities: $1 /|\phi(t)|$ either approaches a finite limit (not greater than $A$ ) or $1 /|\phi(t)|$ approaches zero as $t \rightarrow+\infty$.

In the first case we have from inequality (2)

$$
\left|\alpha(\infty)-\alpha\left(t^{\prime}\right)\right| \leqq \epsilon \quad\left(T \leqq t^{\prime}<\infty\right) .
$$

Since $\epsilon$ is arbitrary and $|1 / \phi(\infty)|$ exists and is not zero,

$$
\left|\alpha(\infty)-\alpha\left(t^{\prime}\right)\right|=o\left|1 / \phi\left(t^{\prime}\right)\right| \text {. }
$$

In the second case for $\left(T \leqq t^{\prime}<\infty\right)$

$$
\begin{aligned}
\left|\alpha(\infty)-\alpha\left(t^{\prime}\right)\right| & <\left|\frac{L(\infty)-L\left(t^{\prime}\right)}{\phi\left(t^{\prime}\right)}\right|+\int_{t^{\prime}}^{\infty}\left|\frac{\phi^{\prime}(u)}{\phi(u)^{2}}\right|[L(u)-L(\infty)\rfloor d u \\
& \leqq \frac{2 \epsilon}{3 A+2}\left|\frac{1}{\phi\left(t^{\prime}\right)}\right| \leqq \epsilon\left|\frac{1}{\phi\left(t^{\prime}\right)}\right| .
\end{aligned}
$$

Since $\epsilon$ is arbitrary our result follows.

17. The behaviour at infinity of certain convolutions. The result which follows is stated in what may seem a rather unnatural form, in order that it may be more immediately applicable later.

THEOREM 17. Let $G(t)$ and $\phi(t)$ be continuous and non-negative $(-\infty<t$ $<\infty)$. If

1a. $\phi(t) \in \downarrow$ for $|t| \geqq T$, for some $T$;

2a. $\phi(+\infty)=0$;

3a. $G\left(t_{0}-t\right)=O\left[\phi(t)\left(t^{2}+1\right)\right]^{-1}$ as $t \rightarrow \pm \infty$, for some $t_{0}$, then

$$
\text { A. } \lim _{x \rightarrow+\infty} \int_{-\infty}^{\infty} G(x-t) \phi(t) d t=0 \text {. }
$$

If $1 \mathrm{~b} . \phi(t) \in \downarrow$

$$
(-\infty<t<T) \text {, for some } T \text {; }
$$

2b. $\phi(+\infty)=0$; 3b. $\left.\begin{array}{rlrl}G\left(t_{0}-t\right) & =O\left[\phi(t)\left(t^{2}+1\right)\right]^{-1} & \text { as } & t \rightarrow-\infty \\ & =O\left[1+t^{2}\right]^{-1} & \text { as } & t \rightarrow+\infty\end{array}\right\}$

for some $t_{0}$, 
then

$$
\text { B. } \lim _{x \rightarrow+\infty} \int_{-\infty}^{\infty} G(x-t) \phi(t) d t=0 .
$$

Conclusion $\mathrm{B}$ is easily seen to be included in conclusion $A$, so that we need prove only A. We shall suppose that $\phi(-\infty)=\infty$, and that $\phi(t)$ does not vanish in any neighborhood of $+\infty$. The slight modifications necessary in the other cases are left to the reader. By increasing the values of $\phi(t)$ in some finite interval and leaving $\phi(t)$ unchanged elsewhere we can construct a new function $\psi(t)$ continuous for $(-\infty<t<\infty)$ and such that $\psi(t) \in \downarrow(-\infty<t<\infty)$.

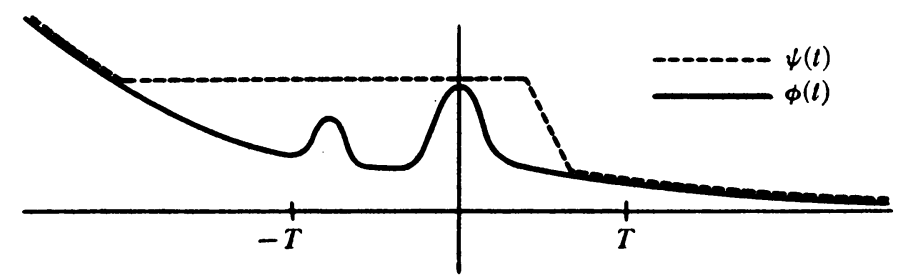

Note that $\phi(t)$ must be modified not just in $(-T \leqq t \leqq T)$ but in a possibly larger interval. We necessarily have

$$
\begin{aligned}
& \phi(t) \leqq \psi(t), \\
& \psi(t) \neq 0 \quad(-\infty<t<\infty),
\end{aligned}
$$

and, since $\phi(t)$ has been modified only over a finite interval,

$$
G\left(t_{0}-t\right)=O\left[\psi(+t)\left(1+t^{2}\right)\right]^{-1} \quad \text { as } t \rightarrow \pm \infty .
$$

Now

$$
\begin{aligned}
\int_{-\infty}^{\infty} G(x-t) \phi(t) d t & \leqq \int_{-\infty}^{\infty} G(x-t) \psi(t) d t \\
& \leqq O(1) \int_{-\infty}^{\infty} \frac{1}{\left(t-x+t_{0}\right)^{2}+1} \frac{\psi(t)}{\psi\left(t-x+t_{0}\right)} d t \\
& <O(1) \int_{-\infty}^{\infty} \frac{1}{y^{2}+1} \frac{\psi\left(y+x-t_{0}\right)}{\psi(y)} d y .
\end{aligned}
$$

For $x \geqq t_{0}$ we see that $\psi\left(y+x-t_{0}\right) / \psi(y) \leqq 1$ since $\psi(t) \in \downarrow$, and further $\lim _{x \rightarrow \infty} \psi\left(y+x-t_{0}\right) / \psi(y)=0$, since $\psi(+\infty)=\phi(+\infty)=0$. By Lebesgue's general convergence theorem

$$
\lim _{x \rightarrow \infty} \int_{-\infty}^{\infty} \frac{1}{\left(y^{2}+1\right)} \frac{\psi\left(y+x-t_{0}\right)}{\psi(y)} d y=0,
$$

and therefore 


$$
\lim _{x \rightarrow \infty} \int_{-\infty}^{\infty} G(x-t) \phi(t) d t=0
$$

as desired.

18. Application of differential operators for class I kernels.

Definition 18a. We set

$$
P_{m}(D)=e^{\left(b-b_{m}\right) D} \prod_{k=1}^{m}\left(1-\frac{D}{a_{k}}\right) e^{D / a_{k}},
$$

where $D$ represents the operation of differentiation and $e^{D / a}$ that of translation through the distance $1 / a$. The variable with respect to which these operations are to be performed will be clear from the context. $P_{m}(D) f(x)$ is defined at $x=x_{0}$ if $f(x) \in C^{n}$ in some interval including in its interior $x_{0}+b-b_{m}+\sum_{k=1}^{m} 1 / a_{k}$. then

As an example, if $f(x) \in C^{2}$ in an interval including $x+b-b_{2}+1 / a_{1}+1 / a_{2}$

$$
\begin{aligned}
P_{2}(D) f(x)=f & \left(x+b-b_{2}+\frac{1}{a_{1}}+\frac{1}{a_{2}}\right) \\
& -\left(\frac{1}{a_{1}}+\frac{1}{a_{2}}\right) f^{\prime}\left(x+b-b_{2}+\frac{1}{a_{1}}+\frac{1}{a_{2}}\right) \\
& +\frac{1}{a_{1} a_{2}} f^{\prime \prime}\left(x+b-b_{2}+\frac{1}{a_{1}}+\frac{1}{a_{2}}\right)
\end{aligned}
$$

LEMMA 18b. If $G(t)$ and $G_{m}(t)$ are defined as in Theorem 3a, and if $P_{m}(D)$ is defined as in Definition 18a, then $P_{m}(D) G(t)=G_{m}(t)(-\infty<t<\infty)$.

Note that we have not assumed here that $G(t)$ is of any class. Since $G(t) \in C^{\infty}(-\infty<t<\infty), P_{m}(D) G(t)$ is certainly defined. We must only verify that it is equal to $G_{m}(t)$. By Theorem $3 \mathrm{a}$,

$$
G(t)=\frac{1}{2 \pi i} \int_{-i \infty}^{i \infty} \frac{e^{s t}}{E(s)} d s .
$$

We wish to apply $P_{m}(D)$ under the integral sign. By a well known general theorem any nonsingular linear differential operator may be applied under an integral sign provided the resulting integral is uniformly convergent. By an elementary computation $P_{m}(D) e^{s t}=P_{m}(s) e^{s t}$. Thus formally

$$
\begin{aligned}
& P_{m}(D) G(t)=\frac{1}{2 \pi i} \int_{-i \infty}^{i \infty} \frac{e^{s t}}{e^{b_{m} s} \prod_{k=m+1}^{\infty}\left(1-\frac{s}{a_{k}}\right) e^{s / a_{k}}} d s \\
& =G_{m}(t) \text {. }
\end{aligned}
$$


By Theorem 2b the integral (1) is uniformly convergent for all $t(-\infty<t<\infty)$, which validates our formal procedure.

LEMMA 18c. If $G(t) \in$ class I and if

$$
f(x)=\int_{-\infty}^{\infty} G(x-t) d \alpha(t)
$$

converges then for $P_{m}(D)$ defined as in Definition 18a

$$
P_{m}(D) f(x)=\int_{-\infty}^{\infty} G_{m}(x-t) d \alpha(t) .
$$

By virtue of Lemma $18 \mathrm{~b}$, and the general theorem quoted in the proof of that lemma, it is enough to show that

$$
\int_{-\infty}^{\infty} G_{m}(x-t) d \alpha(t)
$$

converges uniformly for $x$ in any finite interval. Using the estimates of Theorem 9b, and arguing exactly as in Lemma 15a we may show that

$$
\int_{-\infty}^{\infty}\left|\frac{d}{d t} \frac{G_{m}(x-t)}{G(x-t)}\right| d t
$$

converges uniformly for $x$ in any finite interval. Using Theorem $9 \mathrm{~b}$ we may further show that

$$
\lim _{t \rightarrow+\infty} \frac{G_{m}(x-t)}{G(x-t)} \text { and } \lim _{t \rightarrow-\infty} \frac{G_{m}(x-t)}{G(x-t)}
$$

exist, uniformly for $x$ in any finite interval. If we define

$$
L(t)=\int_{0}^{t} G\left(x_{0}-u\right) d \alpha(u)
$$

for any $x_{0}\left(-\infty<x_{0}<\infty\right)$, then $L(t)$ is bounded and $L(-\infty)$ and $L(+\infty)$ exist. For any $T_{1}$ and $T_{2}$

$$
\begin{aligned}
\int_{T_{v}}^{T_{2}} G_{m}(x-t) d \alpha(t) & =\int_{T_{1}}^{T_{2}} \frac{G_{m}(x-t)}{G(x-t)} d L(t) \\
& =\left[\frac{G_{m}(x-t)}{G(x-t)} L(t)\right]_{T_{1}}^{T_{2}}-\int_{T_{1}}^{T_{2}}\left[\frac{d}{d t} \frac{G_{m}(x-t)}{G(x-t)}\right] L(t) d t .
\end{aligned}
$$

Arguing as in the proof of Theorem 15b, we see that

$$
\int_{-\infty}^{\infty} G_{m}(x-t) d \alpha(t)
$$


is indeed uniformly convergent for $x$ in any finite interval. This proves our lemma.

LEMma 18d. If $G(t) \in$ class I and if

$$
f(x)=\int_{-\infty}^{\infty} G(x-t) \phi(t) d t
$$

converges, then

$$
P_{m}(D) f(x)=\int_{-\infty}^{\infty} G_{m}(x-t) \phi(t) d t .
$$

This is an immediate consequence of Lemma $18 \mathrm{c}$.

LEMMA 18e. If $G(t) \in$ class I and if

$$
f(x)=\int_{-\infty}^{\infty} G(x-t) e^{c t} d \alpha(t)
$$

converges, then:

A. $\alpha_{1}<c<\alpha_{2}$ implies

$$
\begin{aligned}
\alpha(t) & =o\left[e^{\left(\alpha_{2}-c\right) t} t^{-\mu_{2}}\right] & & (\text { as } t \rightarrow+\infty), \\
& =o\left[e^{\left(\alpha_{1}-c\right) t} t^{-\mu_{1}}\right] & & (\text { as } t \rightarrow-\infty) .
\end{aligned}
$$

B. $\alpha_{2} \leqq c$ implies

$$
\begin{aligned}
\alpha(\infty)-\alpha(t) & =o\left[e^{\left(\alpha_{2}-c\right) t} t-\mu_{2}\right] & & (\text { as } t \rightarrow+\infty), \\
\alpha(t) & =o\left[e^{\left(\alpha_{1}-c\right) t} t^{-\mu_{1}}\right] & & (\text { as } t \rightarrow-\infty) .
\end{aligned}
$$

C. $c \leqq \alpha_{1}$ implies

$$
\begin{aligned}
\alpha(t) & =o\left[e^{\left(\alpha_{2}-c\right) t} t^{-\mu_{2}}\right] & & (\text { as } t \rightarrow+\infty), \\
\alpha(t)-\alpha(-\infty) & =o\left[{ }^{\left(\alpha_{1}-c\right) t} t^{\mu_{1}}\right] & & (\text { as } t \rightarrow-\infty) .
\end{aligned}
$$

This lemma is an immediate consequence of Theorem 16. Consider case $\mathrm{B}, t \rightarrow+\infty$, which is typical. By Theorem $9 \mathrm{~b}$

$$
\begin{aligned}
G(x-t) & =q(x-t) e^{\alpha_{2}(x-t)}+O\left[e^{\left(\alpha_{2}+\epsilon\right)(x-t)}\right], \\
\frac{d}{d t} G(x-t) & =\frac{d}{d t}\left[q(x-t) e^{\alpha_{2}(x-t)}\right]+O\left[e^{\left(\alpha_{2}+e\right)(x-t)}\right],
\end{aligned}
$$

as $t \rightarrow+\infty$, where $q(t)$ is a real polynomial of degree $\mu_{2}$ which is positive for $t$ near $-\infty$. If $c>\alpha_{2}$ then these equations together with Leibniz's rule for the differentiation of a product imply that

$$
\frac{d}{d t}\left[e^{c t} G(x-t)\right] \geqq 0
$$


for sufficiently large positive $t$. If $c=\alpha_{2}$, then

$$
\frac{d}{d t}\left[e^{+c t} G(x-t)\right]=\frac{1}{2 \pi i} \int_{-i \infty}^{i \infty} \frac{\alpha_{2}\left(1-s / \alpha_{2}\right) e^{s x-s t+c t}}{E(s)} d s .
$$

Since $\left(1-s / \alpha_{2}\right)$ is a factor of $E(s)$, it may be divided out of numerator and denominator. By Corollary $4 \mathrm{~b}$

$$
\frac{d}{d t}\left[e^{c t} G(x-t)\right] \geqq 0 \quad(-\infty<t<\infty) .
$$

The other hypotheses of Theorem 16 are trivially satisfied so that we now obtain the desired result.

Definition 18f. Let $G(t), G_{m}(t), E(s)$, and $E_{m}(s)$ be defined as in Theorem 3a. $N(G)$ is defined to be the smallest value of $m$, such that $E_{m}(s)$ either does not vanish at $\alpha_{1}$ or has a zero there of multiplicity at least two less than that of $E(s)$ and either does not vanish at $\alpha_{2}$ or has a zero there of multiplicity at least two less than that of $E(s)$. If $\alpha_{1}\left(\right.$ or $\left.\alpha_{2}\right)$ is infinite the requirement is vacuously satisfied at $\alpha_{1}\left(\alpha_{2}\right)$.

This definition applies to kernels of all three classes.

THEOREM 18g. If $G(t) \in$ class $\mathrm{I}$, and if

$$
f(x)=\int_{-\infty}^{\infty} G(x-t) e^{c t} d \alpha(t)
$$

converges, then for $m \geqq N(G), N(G)$ defined as in Definition $18 \mathrm{f}$,

A. $\alpha_{1}<c<\alpha_{2}$ implies

$$
\begin{aligned}
\int_{x_{1}}^{x_{2}} e^{-c x} P_{m}(D) f(x) d x= & \int_{-\infty}^{\infty} G_{m}\left(x_{2}-t\right) e^{-c\left(x_{2}-t\right)} \alpha(t) d t \\
& -\int_{-\infty}^{\infty} G_{m}\left(x_{1}-t\right) e^{-c\left(x_{1}-t\right)} \alpha(t) d t .
\end{aligned}
$$

B. $\alpha_{2} \leqq c$ implies

$$
\int_{x_{1}}^{\infty} e^{-c x} P_{m}(D) f(x) d x=\int_{-\infty}^{\infty} G_{m}\left(x_{1}-t\right) e^{-c\left(x_{1}-t\right)}[\alpha(\infty)-\alpha(t)] d t .
$$

C. $c \leqq \alpha_{1}$ implies

$$
\int_{-\infty}^{x_{2}} e^{-c x} P_{m}(D) f(x) d x=\int_{-\infty}^{\infty} G_{m}\left(x_{2}-t\right) e^{c\left(x_{2}-t\right)}[\alpha(t)-\alpha(-\infty)] d t .
$$

We shall establish B. Conclusions $\mathrm{A}$ and $\mathrm{C}$ follow in similar fashion. By Lemma 18c, we have for every $m$ 


$$
e^{-c x} P_{m}(D) f(x)=\int_{-\infty}^{\infty} G_{m}(x-t) e^{-c(x-t)} d \alpha(t),
$$

the integral converging uniformly for $x$ in any finite interval. Integrating by parts

$$
\begin{aligned}
e^{-c x} P_{m}(D) f(x)= & {\left[G_{m}(x-t) e^{-c(x-t)}[\alpha(t)-\alpha(\infty)]\right]_{-\infty}^{+\infty} } \\
& -\int_{-\infty}^{\infty} \frac{d}{d t}\left[G_{m}(x-t) e^{-c(x-t)}\right][\alpha(t)-\alpha(\infty)] d t
\end{aligned}
$$

By Lemma 18e and Theorem $9 \mathrm{~b}$, the integrated term is zero, uniformly for $x$ in any finite interval. Hence

$$
\begin{aligned}
e^{-c x} P_{m}(D) f(x) & =-\int_{-\infty}^{\infty} \frac{d}{d t}\left[G_{m}(x-t) e^{-c(x-t)}\right][\alpha(t)-\alpha(\infty)] d t \\
& =+\int_{-\infty}^{\infty} \frac{d}{d x}\left[G_{m}(x-t) e^{-c(x-t)}\right][\alpha(t)-\alpha(\infty)] d t
\end{aligned}
$$

this integral converging uniformly for $x$ in any finite interval. We may therefore integrate between finite limits under the sign of integration to obtain

$$
\begin{aligned}
\int_{x_{1}}^{x_{2}} e^{-c x} P_{m}(D) f(x) d x \\
\quad=\int_{-\infty}^{\infty}\left[G_{m}\left(x_{2}-t\right) e^{-c\left(x_{2}-t\right)}-G_{m}\left(x_{1}-t\right) e^{-c\left(x_{1}-t\right)}\right][\alpha(\infty)-\alpha(t)] d t,
\end{aligned}
$$

which for $m \geqq N(G)$ we may write as

$$
\begin{aligned}
\int_{x_{1}}^{x_{2}} e^{-c x} P_{m}(D) f(x) d x= & \int_{-\infty}^{\infty} G_{m}\left(x_{2}-t\right) e^{-c\left(x_{1}-t\right)}[\alpha(\infty)-\alpha(t)] d t \\
& -\int_{-\infty}^{\infty} G_{m}\left(x_{1}-t\right) e^{-c\left(x_{1}-t\right)} \alpha(t) d t
\end{aligned}
$$

We need only show that for $m \geqq N(G)$

$$
\lim _{x_{2} \rightarrow+\infty} \int_{-\infty}^{\infty} G_{m}\left(x_{2}-t\right) e^{-c\left(x_{2}-t\right)}[\alpha(t)-\alpha(\infty)] d t=0 .
$$

This is an immediate consequence of Lemma $18 \mathrm{e}$ and Theorem 17, together with the definition of $N(G)$. Hence, allowing $x_{2}$ to increase without limit in (1) and using (2)

$$
\int_{x_{1}}^{\infty} e^{-c x} P_{m}(D) f(x)=\int_{-\infty}^{\infty} G_{m}\left(x_{1}-t\right) e^{-c\left(x_{1}-t\right)}[\alpha(\infty)-\alpha(t)] d t,
$$


as desired.

19. The inversion theorem for class I kernels.

TheOREM 19a. If $G(t) \in$ class $I$, if the integral

$$
f(x)=\int_{-\infty}^{\infty} G(x-t) e^{-c t} d \alpha(t)
$$

converges, and if $x_{1}$ and $x_{2}$ are points of continuity of $\alpha(t)$ then:

A. $\alpha_{1}<c<\alpha_{2}$ implies

$$
\lim _{m \rightarrow \infty} \int_{x_{1}}^{x_{2}} e^{-c x} P_{m}(D) f(x) d x=\alpha\left(x_{2}\right)-\alpha\left(x_{1}\right) .
$$

B. $\alpha_{2} \leqq c$ implies

$$
\lim _{m \rightarrow \infty} \int_{x_{1}}^{\infty} e^{-c x} P_{m}(D) f(x) d x=\alpha(\infty)-\alpha\left(x_{1}\right) .
$$

C. $c \leqq \alpha_{1}$ implies

$$
\lim _{m \rightarrow \infty} \int_{-\infty}^{x_{2}} e^{-c x} P_{m}(D) f(x) d x=\alpha\left(x_{2}\right)-\alpha(-\infty) .
$$

We shall prove conclusion $\mathrm{B}$. The proofs of $\mathrm{A}$ and $\mathrm{C}$ are similar. $\mathrm{By}$ Theorem $18 \mathrm{~g}$ if $m \geqq N(G)$

$$
\int_{x_{1}}^{\infty} e^{-c x} P_{m}(D) f(x) d x=\int_{-\infty}^{\infty} G_{m}\left(x_{1}-t\right) e^{-c\left(x_{1}-t\right)}[\alpha(\infty)-\alpha(t)] d t
$$

Using Lemma 18e, Theorem 9b, and the definition of $N(G)$, the integral on the right of equation (1) is seen to be absolutely convergent. Let.

$$
\begin{aligned}
I_{m} & =\int_{-\infty}^{\infty} G_{m}\left(x_{1}-t\right) e^{-c\left(x_{1}-t\right)}[\alpha(\infty)-\alpha(t)] d t \\
& =\int_{-\infty}^{\infty} G_{m}(t) e^{-c t}\left[\alpha(\infty)-\alpha\left(x_{1}-t\right)\right] d t .
\end{aligned}
$$

Given $\epsilon>0$ we choose $\delta>0$ so small that

$$
\left|e^{-c t}\left[\alpha(\infty)-\alpha\left(x_{1}-t\right)\right]-\alpha(\infty)+\alpha\left(x_{1}\right)\right| \leqq \epsilon \quad \text { for }|t| \leqq \delta .
$$

We write $I_{m}$ as the sum of three integrals, $I_{m}^{\prime}, I_{m}^{\prime \prime}, I_{m}^{\prime \prime \prime}$, corresponding respectively to the intervals of integration $(-\infty,-\delta),(-\delta, \delta),(\delta, \infty)$.

We first assert that

$$
\lim _{m \rightarrow \infty} I_{m}^{\prime}=\lim _{m \rightarrow \infty} I_{m}^{\prime \prime \prime}=0
$$


By Theorem 11 the function

$$
G_{N(G)}(t)-G_{m}(t)
$$

has at most 2 changes of sign. As $m \rightarrow \infty$ the variance of $G_{m}(t)$ approaches zero. Hence the maximum of $G_{m}(t)$ is becoming larger and larger in the vicinity of the mean of $G_{m}(t)$, which is approaching the origin. This information together with conclusion A of Theorem 13b shows that for $m$ sufficiently large, $m \geqq N_{1}$, the two changes of sign of the function (2) occur within the interval $|t| \leqq \delta$, and

$$
0 \leqq G_{m}(t) \leqq G_{N(G)}(t) . \quad \text { for }|t| \geqq \delta, m \geqq N_{1} .
$$

Hence

$$
\begin{aligned}
\int_{-\infty}^{-\delta} G_{m}(t) e^{-c t}\left[\alpha(\infty)-\alpha\left(x_{1}-t\right)\right] d t & \\
& \ll \int_{-\infty}^{-\delta} G_{N(G)}(t) e^{-c t}\left|\alpha(\infty)-\alpha\left(x_{1}-t\right)\right| d t
\end{aligned}
$$

Using conclusion A of Theorem 13b and Lebesgue's general convergence theorem, we see that

$$
\lim _{m \rightarrow \infty} I_{m}^{\prime}=0
$$

and similarly that

$$
\lim _{m \rightarrow 0} I_{m}^{\prime \prime \prime}=0 .
$$

On the other hand, since $G_{m}(t)$ is non-negative,

$$
\int_{-\delta}^{\delta} G_{m}(t)\left[\alpha(\infty)-\alpha\left(x_{1}\right)-\epsilon\right] d t \leqq I_{m}^{\prime \prime} \leqq \int_{-\delta}^{\delta} G_{m}(t)\left[\alpha(\infty)-\alpha\left(x_{1}\right)+\epsilon\right] d t .
$$

Recalling that $\lim _{m \rightarrow \infty} \int_{-\delta}^{\delta} G_{m}(t) d t=1$, we have

$$
\alpha(\infty)-\alpha\left(x_{1}\right)-\epsilon \leqq \liminf _{m \rightarrow \infty} I_{m} \leqq \limsup _{m \rightarrow \infty} I_{m} \leqq \alpha(\infty)-\alpha\left(x_{1}\right)+\epsilon .
$$

Since $\epsilon$ is arbitrary our theorem follows.

TheOREM 19b. If $G(x) \in$ class $\mathrm{I}$, if

$$
f(x)=\int_{-\infty}^{\infty} G(x-t) \phi(t) d t
$$

converges, and if $x$ is a point of continuity of $\phi(t)$ then

$$
\lim _{m \rightarrow \infty} P_{m}(D) f(x)=\phi(x) .
$$


By Theorem 18c, for any $m$,

$$
P_{m}(D) f(x)=\int_{-\infty}^{\infty} G_{m}(x-t) \phi(t) d t
$$

As before let

$$
\begin{aligned}
I_{m} & =\int_{-\infty}^{\infty} G_{m}(x-t) \phi(t) d t \\
& =\int_{-\infty}^{\infty} G_{m}(t) \phi(x-t) d t .
\end{aligned}
$$

Given $\epsilon>0$, we may choose $\delta$ so small that

$$
|\phi(x-t)-\phi(x)| \leqq \epsilon \quad \text { for }|t| \leqq \delta .
$$

We again express $I_{m}$ as the sum of three integrals $I_{m}^{\prime}, I_{m}^{\prime \prime}, I_{m}^{\prime \prime \prime}$, corresponding to the same intervals of integration as before. We shall show that

$$
\lim _{m \rightarrow \infty} I_{m}^{\prime}=\lim _{m \rightarrow \infty} I_{m}^{\prime \prime \prime}=0
$$

Integrating by parts, we have

$$
I_{m}^{\prime}=\left[\Phi(t) G_{m}(t)\right]_{-\infty}^{-\delta}-\int_{-\infty}^{-\delta} \Phi(t) G_{m}^{\prime}(t) d t
$$

where

$$
\Phi(t)=\int_{-\delta}^{t} \phi(x-u) d u .
$$

By Lemma $18 \mathrm{e}$ and Theorem $9 \mathrm{~b}$ the integrated term vanishes. For $m \geqq N(G)$ the integral so obtained is absolutely convergent, and (using Lemma 18e)

$$
\left|I_{m}^{\prime}\right| \leqq O(1) \int_{-\infty}^{-\delta} e^{-\alpha_{2} t} t^{-\mu_{2}}\left|G_{m}^{\prime}(t)\right| d t
$$

By conclusion B of Theorem 13b, $G_{m}^{\prime}(t)$ is, for $m$ sufficiently large, of constant sign in $(-\infty<t \leqq-\delta)$, so that

$$
\left|I_{m}^{\prime}\right| \leqq O(1)\left|\int_{-\infty}^{-\delta} e^{-\alpha_{2} t-\mu_{2}} G_{m}^{\prime}(t) d t\right| .
$$

Integrating by parts

$$
\left|I_{m}^{\prime}\right| \leqq O(1) G_{m}(-\delta) e^{\alpha_{2} \delta}(-\delta)^{-\mu_{2}}+\int_{-\infty}^{-\delta} G_{m}(t)\left|\left[e^{-\alpha_{2} t} t^{-\mu_{2}}\right]^{\prime}\right| d t
$$


The integrated term approaches zero as $m \rightarrow \infty$ by conclusion $\mathrm{A}$ of Theorem $13 \mathrm{~b}$. The integral converges absolutely for $m \geqq N(G)$, so that by exactly the same argument as was used in the proof of Theorem 19a, it too approaches zero as $m$ approaches infinity. Thus we obtain, as before,

$$
\phi(x)-\epsilon \leqq \liminf _{m \rightarrow \infty} I_{m} \limsup _{m \rightarrow \infty} \leqq \phi(x)+\epsilon
$$

and since $\epsilon$ is arbitrary our theorem follows.

20. An approximate formula for class II kernels. For convolution transforms with class II kernels, our previous methods fail to give a general inversion theorem. The origin of this failure lies in the fact that Theorem $9 \mathrm{~b}$ does not, as it did for class I kernels, yield precise information as to the behaviour of $G(t)$ as $t \rightarrow+\infty$. It tells us only that $G(t)=O\left(e^{-k t}\right)$ as $t \rightarrow \infty$ for every $k$, which leaves open a wide range of possibilities as to the actual behaviour of $G(t)$. In this section we shall derive a formula which will, in our arguments, take the place of Theorem $9 \mathrm{~b}$.

LEMMA 20a. If $G(t) \in$ class II, then the equation

$$
t=\sum_{1}^{\infty} \frac{L}{a_{k}\left(a_{k}+L\right)}
$$

defines $L=L(t)$ as a function of $t$ for $(0 \leqq t<\infty)$. $L(t) \in C^{\infty}, L(t) \in \uparrow$, and $L(+\infty)=\infty$.

Since $G(t) \in$ class II, we have

$$
a_{k}>0, \quad k=1,2, \cdots,
$$

$$
\sum_{1}^{\infty} \frac{1}{a_{k}^{2}}<\infty, \quad \sum_{1}^{\infty} \frac{1}{a_{k}}=\infty .
$$

Differentiating equation (1), a process easily justified by means of equations (2), we obtain

$$
\frac{d t}{d L}=\sum_{1}^{\infty} \frac{1}{\left(a_{k}+L\right)^{2}}
$$

which is positive for $(0 \leqq L<\infty)$. Further as $L$ varies from 0 to $\infty, t$ varies from 0 to $\infty$. Thus by the implicit function theorem $L$ is a function of $t$ for $(0 \leqq t<\infty)$, and evidently $L(t) \in \uparrow, L(\infty)=\infty$. We may obtain any derivative of $L(t)$ by straightforward computation, using of course the fact that

$$
\frac{d t}{d L}>0 \quad(0 \leqq t<\infty)
$$

hence $L(t) \in C^{\infty}$. 
LemMa 20b. If $G(t) \in$ class II then $G(t)$ does not vanish for $(-\infty<t<\infty)$.

Since $G^{\prime}(t)$ has only one change of sign, should $G(t)$ vanish at $t_{0}$, one of the equations $G(t) \equiv 0\left(-\infty<t \leqq t_{0}\right)$ or $G(t) \equiv 0\left(t_{0} \leqq t<\infty\right)$ would hold. The first of these is impossible by Theorem 9b since $\left(\alpha_{2} \neq+\infty\right)$. Thus if $G\left(t_{0}\right)=0$, $G(t) \equiv 0$ for $\left(t_{0}<t<\infty\right)$. We would then have

$$
\int_{-\infty}^{t_{0}} G(t) e^{-s t} d t=\frac{1}{e^{b_{s}} \prod_{1}^{\infty}\left(1-\frac{s}{a_{k}}\right) e^{s / a_{k}}}
$$

which we may rewrite as

$$
\int_{0}^{\infty} G\left(t_{0}-t\right) e^{-s t} d t=\frac{e^{-s t_{0}}}{e^{-b s} \prod_{1}^{\infty}\left(1+\frac{s}{a_{n}}\right) e^{-s / a_{k}}} .
$$

As a Laplace transform the left-hand side of this last equation must approach zero as $s \rightarrow+\infty$. It is easily seen that it does not. Indeed, choose $m$ so large that

$$
b+\sum_{1}^{m} \frac{1}{a_{k}}>t_{0}+1
$$

If $a_{k}>0$ then

$$
\left(1+\frac{s}{a_{k}}\right) e^{-s / a_{k}} \leqq 1 \quad(0 \leqq s<\infty)
$$

It follows that

$$
\frac{e^{-s t_{0}}}{e^{-b s} \prod_{1}^{\infty}\left(1+\frac{s}{a_{k}}\right) e^{-s / a_{k}}} \geqq \frac{e^{+s}}{\prod_{1}^{m}\left(1+\frac{s}{a_{k}}\right)} \quad(0 \leqq s<\infty)
$$

which verifies our assertion.

Let $G(t)$ be a class II kernel. We define, for $r \geqq 0$, the auxiliary kernel $H_{r}(t)$;

$$
H_{r}(t)=\frac{1}{2 \pi i} \int_{-i \infty}^{i \infty} \frac{e^{s t}}{\prod_{1}^{\infty}\left(1-\frac{s}{a_{k}+r}\right) e^{s /\left(a_{k}+r\right)}} d s
$$

It is evident that $H_{r}(t)$ is again a class II kernel. We also set 


$$
\chi(t)=-\log G(t), \quad \chi_{m}(t)=-\log G_{m}(t) .
$$

Using Lemma 20b, we see that $\chi(t)$ and $\chi_{m}(t) \in C^{\infty}$ for $(-\infty<t<\infty)$.

Lemma 20c. If $G(t) \in$ class II, $E(s)$ is defined as in Theorem $3 \mathrm{a}, H_{r}(t)$ is defined by (4), $\chi(t)$ by (5), then

$$
e^{r t-\chi(t)}=\frac{1}{E(-r)} H_{r}\left(t-b-\sum_{k=1}^{\infty} \frac{r}{a_{k}\left(a_{k}+r\right)}\right) \quad(0 \leqq r) .
$$

By Theorem 3a

$$
e^{-x(t)}=\frac{1}{2 \pi i} \int_{-i \infty}^{i \infty} \frac{e^{s t}}{e^{b s} \prod_{1}^{\infty}\left(1-\frac{s}{a_{k}}\right) e^{s / a_{k}}} d s .
$$

Multiplying both sides by $e^{r t}$,

$$
e^{r t-\chi(t)}=\frac{1}{2 \pi i} \int_{-i \infty}^{i \infty} \frac{e^{(\theta+r) t}}{e^{b s} \prod_{1}^{\infty}\left(1-\frac{s}{a_{k}}\right) e^{s / a_{k}}} d s .
$$

Making the change of variable $s+r=u$, and using the identities

$$
\left(1+\frac{r}{a_{k}}\right)\left(1-\frac{u}{r+a_{k}}\right)=1-\frac{u-r}{a_{k}}, \quad \frac{r}{a_{k}\left(a_{k}+r\right)}=\frac{1}{a_{k}}-\frac{1}{r+a_{k}},
$$

this becomes

$$
e^{r t-X(t)}=\frac{1}{2 \pi i} \int_{r-i \infty}^{r+i \infty} \frac{\exp \left(u\left[t-b-\sum^{\infty} \frac{r}{a_{k}\left(a_{k}+r\right)}\right]\right)}{\exp (b r) \prod_{1}^{\infty}\left(1+\frac{r}{a_{k}}\right) \exp \left(-\frac{r}{a_{k}}\right) \prod_{1}^{\infty}\left[1-\frac{u}{r+a_{k}}\right] \exp \left(\frac{u}{r+a_{k}}\right)} d u .
$$

By Theorem $2 b$ and Cauchy's theorem the line of integration may be deformed to the imaginary axis. Recalling the definitions of the various functions under consideration we have

$$
e^{r t-\chi(t)}=\frac{1}{E(-r)} H_{r}\left[t-b-\sum_{1}^{\infty} \frac{r}{a_{k}\left(a_{k}+r\right)}\right]
$$

as desired.

LeMma 20d. If $H_{r}(t)$ is defined as in (4) and if $\zeta_{r}$ is the (by Theorem 13a) unique point associated with the one change of sign of $H_{r}^{\prime}(t)$, then $\zeta_{r}$ is a continuous function of $r$.

It is evident from the definition of $H_{r}(t)$, that, for any fixed $t, H_{r}^{\prime}(t)$ is a 
continuous function of $r$. If our lemma is not true then there exists an $r_{0} \geqq 0$, a sequence $\left\{r_{i}\right\}_{i=1}^{\infty}$ converging to $r_{0}$, and a $\delta>0$, such that

$$
\left|\zeta_{r_{i}}-\zeta_{r_{u}}\right|>\delta, \quad i=1,2, \cdots \text {. }
$$

In this sequence there will be either an infinite number of terms such that

$$
\zeta_{r_{i}}<\zeta_{r_{0}}-\delta
$$

or an infinite number such that

$$
\zeta_{r_{i}}>\zeta_{r_{0}}+\delta,
$$

or both. Suppose for definiteness that equation (6) holds for infinitely many $i$. For notational simplicity we shall suppose it holds for all $i$. By Theorem 13a if $t_{0}$ is any point of the interval $\zeta_{r_{i}}-\delta<t<\zeta_{r_{0}}$, we have

$$
H_{r_{0}}^{\prime}\left(t_{0}\right)>0 \text {. }
$$

On the other hand, it follows from the definition of the $r_{i}$ that

$$
H_{r_{i}}^{\prime}\left(t_{0}\right)<0, \quad i=1,2, \cdots .
$$

Since

$$
\lim _{i \rightarrow \infty} H_{r_{i}}^{\prime}\left(t_{0}\right)=H_{r_{0}}^{\prime}\left(t_{0}\right),
$$

we have thus a contradiction. If we had assumed instead that equation (7) held for an infinite number of $i$, we would have been led to a similar contradiction. Our lemma follows.

Theorem 20e. If $G(t) \in$ class II, $L(t)$ is defined as in Lemma 20a, and $\chi(t)$ is defined as in (5), then

$$
x^{\prime}(t)=L(t-b+o(1))
$$

as $t \rightarrow+\infty$,

where o(1) here represents a function of $t$ which approaches zero as $t$ becomes infinite.

By Lemma 20c,

$$
e^{r t-\chi(t)}=\frac{1}{E(-r)} H_{r}\left[t-b-\sum_{k=1}^{\infty} \frac{r}{a_{k}\left(a_{k}+r\right)}\right] .
$$

Differentiating with respect to $t$ this becomes

$$
\left[r-\chi^{\prime}(t)\right] e^{r t-\chi(t)}=\frac{1}{E(-r)} H_{r}^{\prime}\left[t-b-\sum_{k=1}^{\infty} \frac{r}{a_{k}\left(a_{k}+r\right)}\right] .
$$

If $\zeta_{r}$ is, as before, the point associated with the one change of sign of $H_{r}^{\prime}(t)$, and if in equation (8) we set 


$$
t=b+\sum_{k=1}^{\infty} \frac{r}{a_{k}\left(a_{k}+r\right)}+\zeta_{r}
$$

then we obtain

$$
\chi^{\prime}\left[b+\sum_{k=1}^{\infty} \frac{r}{a_{k}\left(a_{k}+r\right)}+\zeta_{r}\right]=r .
$$

As $r \rightarrow \infty$ the variance of $H_{r}(t)$,

$$
\sum_{1}^{\infty} \frac{1}{\left(a_{k}+r\right)^{2}}
$$

approaches zero, and so by conclusion B of Theorem $13 \mathrm{~b}$, with $b_{m}=0$ and $a_{k}$ replaced by $a_{k}+r$, we see that $\zeta_{r} \rightarrow 0$, as $r \rightarrow \infty$. It approaches zero continuously by virtue of Lemma 20d. Therefore as $r$ varies from zero to infinity all values of $t$ from a certain point onward, say $t \geqq T$, are expressible in the form

$$
t=b+\sum_{k=1}^{\infty} \frac{r}{a_{k}\left(a_{k}+r\right)}+\zeta_{r}
$$

This may conceivably be possible in several ways. For each $t \geqq T$ let $r(t)$ be some one solution of (10). Conceivably $r(t)$ is not a continuous function of $t$. It is clear that as $t \rightarrow+\infty, r(t) \rightarrow+\infty$. Equation (9) may now be rewritten as

$$
\chi^{\prime}[t]=L\left[t-b-\zeta_{r(\ell)}\right] \text {, }
$$

for $t \geqq T$.

Since $r(t) \rightarrow \infty$ as $t \rightarrow \infty, \zeta_{r(t)} \rightarrow 0$ as $t \rightarrow \infty$. If for $\zeta_{r(t)}$ we write $o(1)$, we have our theorem.

TheOREM 20f. If $G(t) \in$ class II, $L(t)$ is defined as in Lemma 20a, and $\chi_{m}(t)$ is defined as in (5), then

$$
\chi_{m}^{\prime}(t)=L\left[t-b_{m}+\sum_{1}^{m} \frac{1}{a_{k}}+o(1)\right] \quad \text { as } t \rightarrow \infty,
$$

where o(1) here represents a function of $t$ which approaches zero as $t \rightarrow \infty$.

If as in Lemma 20a, we define a function $L_{m}(t)$ by means of the equation

$$
t=\sum_{m+1}^{\infty} \frac{L_{m}}{a_{k}\left(a_{k}+L_{m}\right)}
$$

then by Theorem $20 \mathrm{e}$

$$
\chi_{m}^{\prime}(t)=L_{m}\left[t-b_{m}+o(1)\right] \quad \text { as } t \rightarrow+\infty \text {. }
$$

Now 


$$
t+\sum_{1}^{m} \frac{L_{m}}{a_{k}\left(a_{k}+L_{m}\right)}=\sum_{1}^{\infty} \frac{L_{m}}{a_{k}\left(a_{k}+L_{m}\right)}
$$

hence

$$
L_{m}(t)=L\left[t+\sum_{1}^{m} \frac{L_{m}}{a_{k}\left(a_{k}+L_{m}\right)}\right] .
$$

As $t \rightarrow \infty$ we also have $L_{m}(t) \rightarrow \infty$; hence

$$
\sum_{1}^{m} \frac{L_{m}}{a_{k}\left(a_{k}+L_{m}\right)}=\sum_{1}^{m} \frac{1}{a_{k}}+o(1) \quad \text { as } t \rightarrow \infty .
$$

Combining (11), (12) and (13)

$$
\chi_{m}^{\prime}(t)=L\left[t-b_{m}+\sum_{1}^{m} \frac{1}{a_{k}}+o(1)\right]
$$

as desired.

LEMMA 20g. If $H_{r}(t)$ is defined as in (4), and if $\zeta_{r}^{(1)}$ is the (by Theorem 13a) unique point associated with the first change of sign (proceeding from left to right) of $H_{r}^{\prime \prime}(t)$, then $\zeta_{r}^{(1)}$ is a continuous function of $r$.

The proof of this lemma, which resembles the demonstration of Lemma $20 \mathrm{~d}$, is left to the reader.

THEOREM 20h. If $\chi(t)$ is defined as in (5) then for $t$ sufficiently large $(t \geqq T)$ we have

$$
\chi^{\prime \prime}(t) \geqq 0 \text {. }
$$

Differentiating twice the identity of Lemma 20c, we obtain

$$
\left\{-\chi^{\prime \prime}(t)+\left[r-\chi^{\prime}(t)\right]^{2}\right\} e^{r t-x(t)}=\frac{1}{E(-r)} H_{r}^{\prime \prime}\left(t-b-\sum_{k=1}^{\infty} \frac{r}{a_{k}\left(a_{k}+r\right)}\right) \text {. }
$$

If we now set

$$
t=t(r)=b+\sum_{k=1}^{\infty} \frac{r}{a_{k}\left(a_{k}+r\right)}+\zeta_{r}^{(1)} \quad(0 \leqq r<\infty),
$$

where $\zeta_{1}^{(r)}$ is defined as in Lemma $20 \mathrm{~g}$, we obtain

$$
\chi^{\prime \prime}[t(r)]=[r-\chi[t(r)]]^{2} \geqq 0 .
$$

Now $\sum_{k=1}^{\infty} 1 / a_{k}\left(a_{k}+r\right)$ approaches infinity continuously as $r$ approaches infinity, and $\zeta_{r}^{(1)}$ approaches zero continuously (by Theorem $13 \mathrm{~b}$ and Lemma $20 \mathrm{~g}$ ) as $r$ approaches infinity, so that every value of $t$ from a certain point on may be represented in this form 


$$
t=b+\sum_{k=1}^{\infty} \frac{r}{a_{k}\left(a_{k}+r\right)}+\zeta_{r}^{(1)} .
$$

This proves our theorem.

21. Convergence properties of class II kernels.

LEMмa 21a. If $G(t) \in$ class II, and if $\chi(t)$ is defined as in $[5, \S 20]$, then

$$
\lim _{t \rightarrow+\infty} \chi^{\prime}(t) / t=\infty \text {. }
$$

Since by Theorem $20 \mathrm{e}$

$$
\chi^{\prime}(t)=L[t-b+o(1)]
$$

it is sufficient to show that

$$
\lim _{k \rightarrow \infty} L(t) / t=\infty
$$

From the definition of $L(t)$

$$
\frac{d L}{d t}=\left[\sum_{k=1}^{\infty} \frac{1}{\left(a_{k}+L\right)^{2}}\right]^{-1} .
$$

As $t \rightarrow \infty, L \rightarrow \infty$ and $\left[\sum_{k=1}^{\infty}\left(a_{k}+L\right)^{-2}\right]^{-1} \rightarrow+\infty$. Equation (1), and therefore our lemma, follows immediately.

LEMMA 21b. If $G(t) \in$ class II, then

$$
\text { A. } \lim _{t \rightarrow-\infty} \frac{G(x-t)}{G\left(x_{0}-t\right)}=0
$$

uniformly for $\left(x_{1} \leqq x<\infty\right)$ for any $x_{1}>x_{0}$.

$$
\text { B. } \int_{-\infty}^{0}\left|\frac{G^{\prime}(x-t)}{G\left(x_{0}-t\right)}\right| d t
$$

converges uniformly for $\left(x_{1} \leqq x<\infty\right)$ for any $x_{1}>x_{0}$.

We have

$$
\frac{G(x-t)}{G\left(x_{0}-t\right)}=e^{+\chi\left(x_{0}-t\right)-\chi(x-t)} .
$$

Using the mean value theorem we obtain

$$
\left.\chi(x-t)-\chi\left(x_{0}-t\right)\right]=\left(x-x_{0}\right) \chi^{\prime}\left[x_{0}-t+\theta\left(x-x_{0}\right)\right],
$$

where $0<\theta<1$. Now

$$
\chi^{\prime}(t) \geqq 0
$$


for $t \geqq \zeta$ where $\zeta$ is the one change of sign of $G^{\prime}(t)$. Also for $t$ sufficiently large $\left(t \geqq T_{0}\right)$, we know by Theorem $20 \mathrm{~h}$ that

$$
\chi^{\prime \prime}(t) \geqq 0 \text {. }
$$

Thus if $T=\max \left(T_{0}, \zeta\right)-x_{0}$, we have

$$
\begin{aligned}
\chi(x-t)-\chi\left(x_{0}-t\right) & \geqq\left(x-x_{0}\right) \chi^{\prime}\left(x_{0}-t\right) \\
& \geqq\left(x_{1}-x_{0}\right) \chi^{\prime}\left(x_{0}-t\right),
\end{aligned}
$$

for all $t$ such that $t \leqq-T$ and all $x$ such that $x_{1} \leqq x<\infty$. Hence

$$
0 \leqq \frac{G(x-t)}{G\left(x_{0}-t\right)} \leqq e^{-\left(x_{1}-x_{0}\right) x^{\prime}\left(x_{0}-t\right)} \quad\left(t \leqq-T ; x_{1} \leqq x<\infty\right) .
$$

Applying Lemma 21a we obtain conclusion A.

Consider next the integral

$$
\int_{\infty}^{-T} \chi^{\prime}\left(x_{0}-t\right) e^{x\left(x_{0}-t\right)-x(x-t)} d t
$$

By virtue of the estimates just made we have

$$
0 \leqq \chi^{\prime}\left(x_{0}-t\right) e^{\chi\left(x_{0}-t\right)-\chi(x-t)} \leqq \chi^{\prime}\left(x_{0}-t\right) e^{-\left(x_{1}-x_{0}\right) \chi^{\prime}\left(x_{0}-t\right)},
$$

from which, using the inequality

$$
x e^{-x}<e^{-x / 2} \quad(0 \leqq x<\infty),
$$

we obtain

$$
0 \leqq \chi^{\prime}\left(x_{0}-t\right) e^{\chi\left(x_{0}-t\right)-\chi(x-t)} \leqq e^{-\left(x_{1}-x_{0}\right) \chi^{\prime}\left(x_{0}-t\right) / 2} .
$$

Using Lemma 21a, the uniform convergence of the integral (2) follows.

Integrating the integral (2) by parts we obtain

$$
\int_{-L}^{-T} \chi^{\prime}\left(x_{0}-t\right) e^{x\left(x_{0}-t\right)-\chi(x-t)}=\left[-\frac{G(x-t)}{G\left(x_{0}-t\right)}\right]_{-L}^{-T}-\int_{-L}^{-T} \frac{G^{\prime}(x-t)}{G\left(x_{0}-t\right)} d t .
$$

Conclusion A and the uniform convergence of the integral (2) thus imply the uniform convergence of the integral

$$
\int_{-\infty}^{-T} \frac{G^{\prime}(x-t)}{G\left(x_{0}-t\right)} d t
$$

Since $G^{\prime}(x-t) / G(x-t)$ is of constant (negative) sign for $t \leqq-T$ and $\left(x_{1} \leqq x<\infty\right)$, the integral

$$
\int_{-\infty}^{-T}\left|\frac{G^{\prime}(x-t)}{G\left(x_{0}-t\right)}\right| d t
$$


and finally the integral

$$
\int_{-\infty}^{0}\left|\frac{G^{\prime}(x-t)}{G\left(x_{0}-t\right)}\right| d t
$$

are uniformly convergent.

THEOREM 21c. If $G(t) \in$ class II, and if the transform

$$
f(x)=\int_{-\infty}^{\infty} G(x-t) d \alpha(t)
$$

converges for $x_{0}$ it converges for all $x>x_{0}$, and if $x_{1}>x_{0}$ it converges uniformly for $x_{1} \leqq x \leqq x_{2}$.

Since the behaviour of $G(x-t)$ as $t \rightarrow+\infty$ is, by Theorem $9 \mathrm{~b}$, like that of a class I kernel, the convergence of (3) for any $x$ implies (as in the proof of Theorem 15b) the convergence of

$$
\int_{0}^{\infty} G(x-t) d \alpha(t)
$$

for all $x$, uniformly for $x$ in any finite interval.

Now

$$
\int_{-L}^{0} G(x-t) d \alpha(t)=[G(x-t) \alpha(t)]_{-L}^{0}+\int_{-L}^{0} \alpha(t) G^{\prime}(x-t) d t
$$

The convergence of (3) for $x=x_{0}$ implies, by virtue of Theorem 16 (part A), that

$$
\alpha(t)=o\left[G\left(x_{0}-t\right)\right]^{-1}
$$
as $t \rightarrow-\infty$.

Hence

$$
|G(x-L) \alpha(L)| \leqq O(1) \frac{G(x-L)}{G\left(x_{0}-L\right)},
$$

and

$$
\int_{-L}^{0}\left|\alpha(t) G^{\prime}(x-t)\right| d t \leqq O(1) \int_{-L}^{0}\left|\frac{G^{\prime}(x-t)}{G\left(x_{0}-t\right)}\right| d t
$$

The uniform convergence of the integral (3) for $x_{1} \leqq x<\infty$ now follows from Lemma $21 \mathrm{~b}$. Thus our theorem is proved.

COROLLARY 21d. If $G(t) \in$ class II and if the transform

$$
f(x)=\int_{-\infty}^{\infty} G(x-t) \phi(t) d t
$$


converges for $x_{0}$ it converges for all $x>x_{0}$ and if $x_{1}>x_{0}$ it converges uniformly for $x_{1} \leqq x \leqq x_{2}$.

As a consequence of these two theorems we see that if $G(t) \in$ class II there exists a constant $\gamma_{c}$, which may be $+\infty$ or $-\infty$, depending on $\alpha(t)$ or $\phi(t)$ such that the transform

$$
\int_{-\infty}^{\infty} G(x-t) d \alpha(t) \text { or } \int_{-\infty}^{\infty} G(x-t) \phi(t) d t
$$

converges for $x>\gamma_{c}$ and diverges for $x<\gamma_{c}$. For $x=\gamma_{c}$ it may converge or diverge. This number $\gamma_{c}$ is called the abscissa of convergence of the transform.

22. Application of the differential operator for class II kernels.

LEMMA 22a. If $G(t) \in$ class II and if $\chi(t)$ and $\chi_{m}(t)$ are defined as in $[5, \S 20]$ then given any $\epsilon>0$, there are constants $T$ and $C$ such that

$$
\chi_{m}(t) \geqq \chi\left(t+b-b_{m}+\sum_{1}^{m} \frac{1}{a_{k}}-\epsilon\right)+C \quad(T \leqq t<\infty) .
$$

By integrating the estimates of Theorems $20 \mathrm{e}$ and $20 \mathrm{f}$ and using the monotonic character of $L(t)$ one obtains this result.

LEMMA 22b. If $G(t) \in$ class II, then

$$
\text { A. } \lim _{t \rightarrow-\infty} \frac{G_{m}(x-t)}{G\left(x_{0}-t\right)}=0,
$$

uniformly for $\left(x_{1} \leqq x<\infty\right)$ for any $x_{1}>x_{0}+b_{m}-b-\sum_{k=1}^{m} 1 / a_{k}$;

$$
\text { B. } \int_{-\infty}^{0}\left|\frac{G_{m}^{\prime}(x-t)}{G_{0}(x-t)}\right| d t
$$

converges uniformly for $\left(x_{1} \leqq x<\infty\right)$ for any $x_{1}>x_{0}+b_{m}-b-\sum_{k=1}^{m} 1 / a_{k}$.

Choose any positive $\epsilon<x_{1}-x_{0}-b_{m}+b+\sum_{k=1}^{m} 1 / a_{k}$. Then by Lemma 22a there will exist constants $T$ and $C$ such that

$$
G_{m}(x-t)<e^{c} G\left(x-t+b-b_{m}+\sum_{1}^{m} \frac{1}{a_{k}}-\epsilon\right) \quad(t \geqq T),
$$

from which we obtain

$$
\frac{G_{m}(x-t)}{G_{0}(x-t)} \leqq e^{c} \frac{G\left(x-t+b-b_{m}+\sum_{1}^{m} \frac{1}{a_{k}}-\epsilon\right)}{G\left(x_{0}-t\right)} .
$$

Since $x \geqq x_{1}>x_{0}+b_{m}-b-\sum_{k=1}^{m} 1 / a_{k}$ 


$$
x-t+b-b_{m}+\sum_{1}^{m} \frac{1}{a_{k}}-\epsilon>x_{0}-t+\delta,
$$

where

$$
\delta=x_{1}-x_{0}-b_{m}+b+\sum_{1}^{m} \frac{1}{a_{k}}-\epsilon>0 .
$$

Conclusion A now follows from conclusion A of Lemma 21a. This estimate may be again applied to show, after the proof of Lemma 21a, that

$$
\int_{-\infty}^{0} x^{\prime}\left(x_{0}-t\right) e^{x\left(x_{0}-t\right)-x_{m}(x-t)} d t
$$

is uniformly convergent for $x_{1} \leqq x<\infty$. Integrating this by parts and using conclusion A we obtain conclusion $\mathrm{B}$, on noting that $G_{m}^{\prime}(x-t) / G\left(x_{0}-t\right)$ is of constant sign for $t \leqq-T_{1}$ and for all $x \geqq x_{1}$, where $T_{1}$ is an appropriate constant.

THEOREM 22c. If $G(t) \in$ class II, and if the transform

$$
f(x)=\int_{-\infty}^{\infty} G(x-t) d \alpha(t)
$$

has abscissa of convergence $\gamma_{c}$ then

$$
P_{m}(D) f(x)=\int_{-\infty}^{\infty} G_{m}(x-t) d \alpha(t),
$$

the integral converging uniformly for $\left(x_{1} \leqq x \leqq x_{2}\right)$ for any $x_{1}$ and $x_{2}$ such that $\gamma_{c}+b_{m}-b-\sum_{1}^{m} 1 / a_{k}<x_{1}$.

It is clearly sufficient to prove that the integral (2) is uniformly convergent for $\left(x_{1} \leqq x \leqq x_{2}\right)$. Since, as $t \rightarrow+\infty, G(x-t)$ behaves like a class I kernel, the convergence of (1) for any value of $x$ implies the uniform convergence of

$$
\int_{0}^{\infty} G_{m}(x-t) d \alpha(t)
$$

for $x$ in any finite interval.

By the definition of $\gamma_{0}$ there must be a value $x_{0}$

$$
x_{0}-\gamma_{c}<x_{1}-\gamma_{c}-b_{m}+b+\sum_{1}^{m} \frac{1}{a_{k}}
$$

for which (1) converges. By Theorem 16 (part A)

$$
\alpha(t)=o\left[G\left(x_{0}-t\right)\right]^{-1} \quad \text { as } t \rightarrow-\infty .
$$


Integrating by parts, we have

$$
\int_{-T}^{0} G_{m}(x-t) d \alpha(t)=\left[G_{m}(x-t) \alpha(t)\right]_{-T}^{0}+\int_{-T}^{0} G_{m}^{\prime}(x-t) \alpha(t) d i
$$

Using the estimate (3) and Lemma 22b we see that the integral

$$
\int_{\infty}^{0} G_{m}(x-t) d \alpha(t)
$$

is uniformly convergent for $\left(x_{1} \leqq x<\infty\right)$. Thus our lemma is proved.

THEOREM 22d. If $G(t) \in$ class II and if the transform

$$
f(x)=\int_{-\infty}^{\infty} G(x-t) \phi(t) d t
$$

has abscissa of convergence $\gamma_{c}$, then

$$
P_{m}(D) f(x)=\int_{-\infty}^{\infty} G_{m}(x-t) \phi(t) d t,
$$

the integral converging uniformly for $\left(x_{1} \leqq x \leqq x_{2}\right)$ for any $x_{1}$ and $x_{2}$ such that

$$
\gamma_{c}+b_{m}-b-\sum_{1}^{m} \frac{1}{a_{k}}<x_{1} .
$$

This is a corollary of Theorem 22c.

THEOREM 22e. If $G(t) \in$ class II, if the transform

$$
f(x)=\int_{-\infty}^{\infty} G(x-t) e^{c t} d \alpha(t)
$$

has abscissa of convergence $\gamma_{c}$, and if $\gamma_{c}+b_{m}-b-\sum_{1}^{m} 1 / a_{k}<x_{1}<x_{2}$, then

A. $c<\alpha_{2}$ and $m \geqq N(G)$ (see Definition 18f) imply that

$$
\begin{aligned}
\int_{x_{1}}^{x_{2}} e^{-c x} P_{m}(D) f(x) d x= & \int_{-\infty}^{\infty} G_{m}\left(x_{2}-t\right) e^{-c\left(x_{2}-t\right)} \alpha(t) d t \\
& -\int_{-\infty}^{\infty} G_{m}\left(x_{1}-t\right) e^{-c\left(x_{1}-t\right)} \alpha(t) d t ;
\end{aligned}
$$

B. $\alpha_{2} \leqq c$ and $m \geqq N(G)$ (see Definition 18f) imply that $\alpha(+\infty)$ exists and that

$$
\int_{x_{1}}^{\infty} e^{-c x} P_{m}(D) f(x) d x=\int_{-\infty}^{\infty} G_{m}\left(x_{1}-t\right) e^{-c\left(x_{1}-t\right)}[\alpha(\infty)-\alpha(t)] d t .
$$

Case A. By Theorem 22c, the integral 


$$
\int_{-\infty}^{\infty} G_{m}(x-t) e^{-c(x-t)} d \alpha(t)=e^{-c x} P_{m}(D) f(x)
$$

converges uniformly for $\left(x_{1} \leqq x \leqq x_{2}\right)$. Further by Theorem 16 , and so on,

$$
\lim _{t \rightarrow \pm \infty} G(x-t) e^{-c(x-t)} \alpha(t)=0
$$

uniformly for $\left(x_{1} \leqq x \leqq x_{2}\right)$. Hence we may integrate (4) by parts to obtain

$$
-\int_{-\infty}^{\infty} \frac{d}{d t}\left[G_{m}(x-t) e^{-c(x-t)}\right] \alpha(t) d t=e^{-c x} P_{m}(D) f(x),
$$

which is uniformly convergent for $\left(x_{1} \leqq x \leqq x_{2}\right)$. This may be rewritten as

$$
\int_{-\infty}^{\infty} \frac{d}{d x}\left[G_{m}(x-t) e^{-c(x-t)}\right] \alpha(t) d t=e^{-c x} P_{m}(D) f(x) .
$$

If we integrate under the integral sign between the limits $x_{1}$ and $x_{2}$, which is permissible because of the uniform convergence, we obtain

$$
\int_{x_{1}}^{x_{2}} e^{-c x} P_{m}(D) f(x) d x=\int_{-\infty}^{\infty}\left[G_{m}\left(x_{2}-t\right) e^{-c\left(x_{2}-t\right)}-G_{m}\left(x_{1}-t\right) e^{-c\left(x_{1}-t\right)}\right] \alpha(t) d t .
$$

If $m \geqq N(G)$ this may be written as

$$
\begin{aligned}
\int_{x_{1}}^{x_{2}} e^{-c x} P_{m}(D) f(x) d x= & \int_{-\infty}^{\infty} G_{m}\left(x_{2}-t\right) e^{-c\left(x_{2}-t\right)} \alpha(t) d t \\
& -\int_{-\infty}^{\infty} G_{m}\left(x_{1}-t\right) e^{-c\left(x_{1}-t\right)} \alpha(t) d t,
\end{aligned}
$$

since for $m \geqq N(G)$ each of these integrals is (absolutely) convergent.

Case B. By Theorem 22c, the integral

$$
e^{-c x} P_{m}(D) f(x) d x=\int_{-\infty}^{\infty} G_{m}(x-t) e^{-c(x-t)} d \alpha(t)
$$

converges uniformly for $\left(x_{1} \leqq x \leqq x_{2}\right)$ for every $x_{2} \geqq x_{1}$. By Theorem 16 (part B)

$$
\lim _{t \rightarrow \pm \infty} G(x-t) e^{-c(x-t)}[\alpha(\infty)-\alpha(t)]=0,
$$

uniformly for $\left(x_{1} \leqq x \leqq x_{2}\right)$. Arguing just as in case A we obtain

$$
\begin{aligned}
\int_{x_{1}}^{x_{2}} e^{-c x} P_{m}(D) f(x) d x= & \int_{-\infty}^{\infty} G_{m}\left(x_{2}-t\right) e^{-c\left(x_{2}-t\right)}[\alpha(t)-\alpha(\infty)] d t \\
& +\int_{-\infty}^{\infty} G_{m}\left(x_{1}-t\right) e^{-c\left(x_{1}-t\right)}[\alpha(\infty)-\alpha(t)] d t .
\end{aligned}
$$


We must show that

$$
\lim _{x \rightarrow \infty} \int_{-\infty}^{\infty} G_{m}\left(x_{2}-t\right) e^{-c\left(x_{2}-t\right)}[\alpha(t)-\alpha(\infty)] d t=0 .
$$

Consider the case where $\alpha_{2}<c$. Using the estimates of Theorem 16 we see that

$$
\int_{-\infty}^{\infty} G_{m}\left(x_{2}-t\right) e^{-c\left(x_{2}-t\right)}[\alpha(t)-\alpha(\infty)] d t \ll O(1) \int_{-\infty}^{\infty} G_{m}\left(x_{2}-t\right) e^{-c\left(x_{0}-t\right)} A(t) d t,
$$

where

$$
A(t)=\left[G\left(x_{0}-t\right) e^{-c\left(x_{2}-t\right)}\right]^{-1},
$$

$x_{0}$ being any number greater than $\gamma_{c}$.

We first assert that

$$
\begin{array}{rr}
A(t) \geqq 0 & (-\infty<t<\infty), \\
A(t) \in \downarrow & (|t| \geqq T),
\end{array}
$$

where $T$ is some suitable constant. Inequality (5) follows immediately from the fact that $G(t)>0$. When $t$ is large and positive the asymptotic estimate of Theorem $9 \mathrm{~b}$ may be employed to show that $A(t) \in \downarrow$, and also that $A(+\infty)$ $=0$. When $t$ is large and negative the equation

$$
A^{\prime}(t)=\frac{\left[G^{\prime}\left(x_{0}-t\right)-c G\left(x_{0}-t\right)\right] e^{-c\left(x_{0}-t\right)}}{\left[G\left(x_{0}-t\right) e^{-c\left(x_{0}-t\right)}\right]^{2}}
$$

shows that $A^{\prime}(t) \leqq 0$, that is, $A(t) \in \downarrow$.

Finally we assert that if

$$
t_{0}>x_{0}+b-b_{m}-\sum_{1}^{m} \frac{1}{a_{k}}
$$

then

$$
G_{m}\left(t_{0}-t\right) e^{-c\left(t_{0}-t\right)}=O\left[\frac{1}{1+t^{2}} G\left(x_{0}-t\right) e^{-c\left(x_{0}-t\right)}\right], \quad t \rightarrow \pm \infty .
$$

When $t$ is large and positive this follows from the asymptotic estimate of Theorem $9 \mathrm{~b}$, and the fact that $m \geqq N(G)$. When $t$ is large and negative equation (8) may be shown to be true by using Lemma 22a, and so on.

The assumptions of Theorem 17 (A) are thus satisfied. It follows that

$$
\lim _{x \rightarrow \infty} \int_{-\infty}^{\infty} G_{m}\left(x_{2}-t\right) e^{-c\left(x_{2}-t\right)} A(t) d t=0
$$


and hence that

$$
\lim _{x_{m} \rightarrow \infty} \int_{-\infty}^{\infty} G_{m}\left(x_{2}-t\right) e^{-c\left(x_{2}-t\right)}[\alpha(t)-\alpha(\infty)]=0,
$$

that is,

$$
\int_{x_{1}}^{\infty} e^{-e x} P_{m}(D) f(x) d x=\int_{-\infty}^{\infty} G_{m}\left(x_{1}-t\right) e^{-c\left(x_{1}-t\right)}[\alpha(\infty)-\alpha(t)] d t .
$$

Thus we have proved conclusion $\mathrm{B}$, under the assumption that $\alpha_{2}<c$. The case where $\alpha_{2}=c$ may be disposed of with only slight variations.

23. The inversion theorem for class II kernels.

TheOREM 23a. If $G(t) \in$ class II, if

$$
f(x)=\int_{-\infty}^{\infty} G(x-t) e^{c t} d \alpha(t)
$$

has abscissa of convergence $\gamma_{c}$, and if $x_{1}$ and $x_{2}$ are points of continuity of $\alpha(t)$ then

A. $c<\alpha_{2}$ implies

$$
\lim _{m \rightarrow \infty} \int_{x_{1}}^{x_{2}} e^{-c x} P_{m}(D) f(x) d x=\alpha\left(x_{2}\right)-\alpha\left(x_{1}\right) .
$$

B. $\alpha_{2} \leqq c$ implies

$$
\lim _{m \rightarrow \infty} \int_{x_{1}}^{\infty} e^{-c x} P_{m}(D) f(x) d x=\alpha(\infty)-\alpha\left(x_{1}\right) .
$$

For all $m$ sufficiently large, $m \geqq M$, we have

$$
x_{2}>x_{1}>\gamma_{c}+b_{m}-b-\sum_{1}^{m} \frac{1}{a_{k}} \text {. }
$$

Thus, by Theorem 22e, we must show that

$$
\lim _{m \rightarrow \infty} \int_{-\infty}^{\infty} G_{m}\left(x_{1}-t\right) e^{-c\left(x_{1}-t\right)} \alpha(t) d t=\alpha\left(x_{1}\right) \quad \text { for } c<\alpha_{2},
$$

and

$$
\begin{aligned}
\lim _{m \rightarrow \infty} \int_{-\infty}^{\infty} G_{m}\left(x_{1}-t\right) e^{-c\left(x_{1}-t\right)}[\alpha(\infty)-\alpha(t)] d t=\alpha(\infty)-\alpha\left(x_{1}\right), & \\
& \text { for } \alpha_{2}<c .
\end{aligned}
$$

For $m=\max [M, N(G)]$, the integrals (1) and (2) are, by familiar arguments, absolutely convergent. The proof is now a verbatim repetition of the proof 
of Theorem 19a. Theorems 11 and $13 \mathrm{~b}$ are used to show that for any $\delta>0$

$$
\lim _{m \rightarrow \infty} \int_{\left|x_{1}-t\right| \geqq \delta} G_{m}\left(x_{1}-t\right) e^{-c\left(x_{1}-t\right)}[\alpha(\infty)-\alpha(t)] d t=0,
$$

and Theorem 12 is then employed in a Fejer type argument to complete the proof.

TheOREM 23b. If $G(t) \in$ class II, if

$$
f(x)=\int_{-\infty}^{\infty} G(x-t) \phi(t) d t
$$

has abscissa of convergence $\gamma_{c}$ and if $x_{1}$ is a point of continuity of $\phi(t)$ then

$$
\lim _{m \rightarrow \infty} P_{m}(D) f\left(x_{1}\right)=\phi\left(x_{1}\right) \text {. }
$$

For all $m$ sufficiently large we have

$$
x_{1}>\gamma_{c}+b_{m}-b-\sum_{1}^{m} \frac{1}{a_{k}} ;
$$

hence by Theorem $22 \mathrm{~d}$ it is enough to prove that

$$
\lim _{m \rightarrow \infty} \int_{-\infty}^{\infty} G_{m}\left(x_{1}-t\right) \phi(t) d t=\phi\left(x_{1}\right)
$$

The difficult point is to show that, given $\delta>0$,

$$
\begin{aligned}
& \lim _{m \rightarrow \infty} \int_{x_{1}+\delta}^{\infty} G_{m}\left(x_{1}-t\right) \phi(t) d t=0, \\
& \lim _{m \rightarrow \infty} \int_{-\infty}^{x_{1}-\delta} G_{m}\left(x_{1}-t\right) \phi(t) d t=0 .
\end{aligned}
$$

In order to apply Theorem 11 these integrals must be transformed so that they become absolutely convergent. As $t \rightarrow+\infty, G(x-t)$ behaves like a class I kernel, and the demonstration of (3) may be taken bodily from the proof of Theorem 19b. To establish (4) we integrate by parts obtaining

$$
\int_{\delta}^{\infty} G_{m}(t) \phi\left(x_{1}-t\right) d t=\left[G_{m}(t) \psi(t)\right]_{\delta}^{\infty}-\int_{\delta}^{\infty} G_{m}^{\prime}(t) \psi(t) d t,
$$

where

$$
\psi(t)=\int_{\delta}^{t} \phi\left(x_{1}-t\right) d t .
$$

If $x_{0}$ is any number, $x_{0}>\gamma_{c}$, then by Theorem 16 


$$
\psi(t)=o\left[G\left(x_{0}-x_{1}+t\right)\right]^{-1} \quad(t \rightarrow+\infty) .
$$

For $m$ so large that

$$
x_{1}>x_{0}+b_{m}-\sum_{1}^{m} \frac{1}{a_{k}},
$$

the integrated term in equation (5) will vanish and the integral on the right will be absolutely convergent, by Lemma $22 \mathrm{~b}$. This justifies equation (5). We now have

$$
\left|\int_{\delta}^{\infty} G_{m}(t) \phi\left(x_{1}-t\right) d t\right| \leqq O(1) \int_{\delta}^{\infty}\left|e^{x\left(x_{0}-x_{1}+t\right)} G_{m}^{\prime}(t)\right| d t .
$$

For all $m$ sufficiently large $G_{m}^{\prime}(t)$ is, by Theorem $13 \mathrm{~b}$, of constant sign in the interval $(\delta \leqq t<\infty)$, so that

$$
\left|\int_{\delta}^{\infty} G_{m}(t) \phi\left(x_{1}-t\right) d t\right| \leqq O(1)\left|\int_{\delta}^{\infty} e^{x\left(x_{0}-x_{1}+t\right)} G_{m}^{\prime}(t) d t\right| .
$$

Integrating by parts

$$
\begin{aligned}
& \quad\left|\int_{\delta}^{\infty} G_{m}(t) \phi\left(x_{1}-t\right) d t\right| \\
& \quad \leqq O(1) e^{\chi\left(x_{0}-x_{1}+\delta\right)} G_{m}(\delta)+O(1)\left|\int_{\delta}^{\infty} G_{m}(t) \chi^{\prime}\left(x_{0}-x_{1}+t\right) e^{\chi\left(x_{0}-x_{1}+t\right)} d t\right| .
\end{aligned}
$$

By Theorem 13b the integrated term will approach zero as $m \rightarrow \infty$. By Lemma $22 \mathrm{~b}$ the integral on the right is absolutely convergent. Theorem 11, and so on, may now be employed to show that it approaches zero as $m \rightarrow \infty$.

Having established (1) and (2) the proof may easily be completed.

24. The inversion formula for class III kernels. The kernels of this class are like the kernels of class II except that instead of approaching zero very rapidly as $t \rightarrow+\infty$, they simply vanish from a certain point on. This "degeneracy" makes them very easy to discuss, and indeed the methods developed for kernels of class I suffice here.

We previously supposed that $\phi(t)$ (or $\alpha(t))$ was defined for $(-\infty<t<\infty)$ and was integrable (of bounded variation) in every finite subinterval. Here we need merely specify that $\phi(t)$ (or $\alpha(t)$ ) is defined for $\left(t_{0}<t<\infty\right)$, and is integrable (of bounded variation) in every closed finite subinterval. The number $t_{0}$ is a constant, possibly $-\infty$, associated with $\phi(t)$ (or $\left.\alpha(t)\right)$.

LEMma 24a. If $G(t) \in$ class III, then $G(t)$ does not vanish for $(-\infty<t<b$ $\left.+\sum_{1}^{\infty} 1 / a_{k}\right)$.

If our lemma is not true, then there is a number $\xi$, 


$$
\xi<b+\sum_{1}^{\infty} \frac{1}{a_{k}}
$$

such that $G(\xi)=0$. From Theorem $10, G(t)=0$ for $(\xi \leqq t<\infty)$. Now

$$
\int_{-\infty}^{\xi} G(t) e^{-s t} d t=\frac{1}{e^{b s} \prod_{1}^{\infty}\left(1-\frac{s}{a_{k}}\right) e^{s / a_{k}}} .
$$

After a change of variables this becomes

$$
\int_{0}^{\infty} e^{-s y G}\left(b+\sum_{1}^{\infty} 1 / a_{k}-y\right) d y=\frac{1}{\prod_{1}^{\infty}\left(1+\frac{s}{a_{k}}\right)}
$$

where $\epsilon=b-\xi+\sum_{1}^{\infty} 1 / a_{k}$. This implies by the elementary theory of the Laplace transform that

$$
\frac{1}{\prod_{1}^{\infty}\left(1+\frac{s}{a_{k}}\right)}=o\left(e^{-c s}\right)
$$
as $s \rightarrow+\infty$.

Since $\prod_{1}^{\infty}\left(1+\left[s / a_{k}\right]\right)$ is at most of order 1 minimal type, see [2; vol. II, p. 249], this is impossible, and our lemma follows.

Theorem 24b. If $G(t) \in$ class III, if $\alpha(t)$ is defined for $\left(t_{0}<t<\infty\right)$, and if the transform,

$$
\int_{-\infty}^{\infty} G\left(x_{0}-t\right) d \alpha(t)
$$

converges for some $x_{0}>t_{0}+b+\sum_{1}^{\infty} 1 / a_{k}$, then it converges for all $x>t_{0}+b$ $+\sum_{1}^{\infty} 1 / a_{k}$, and uniformly for $\left(x_{1} \leqq x \leqq x_{2}\right)$, where $t_{0}+b+\sum_{1}^{\infty} 1 / a_{k}<x_{1}<x_{2}<\infty$.

The proof is similar to that of Theorem $15 \mathrm{~b}$ and is omitted.

Theorem 24c. If $G(t) \in$ class III, if $\alpha(t)$ is defined for $\left(t_{0}<t<\infty\right)$, if .

$$
f(x)=\int_{-\infty}^{\infty} G(x-t) e^{+c t} d \alpha(t)
$$

converges for some $x>t_{0}+b+\sum_{1}^{\infty} 1 / a_{k}$, and if $x_{1}$ and $x_{2}$ are points of continuity of $\alpha(t), x_{1}, x_{2}>t_{0}$, then

A. $c<\alpha_{1}$ implies

$$
\lim _{m \rightarrow \infty} \int_{x_{1}}^{x_{2}} e^{-c x} P_{m}(D) f(x) d x=\alpha\left(x_{2}\right)-\alpha\left(x_{1}\right)
$$


B. $\alpha_{1} \leqq c$ implies

$$
\lim _{m \rightarrow \infty} \int_{x_{1}}^{\infty} e^{-c x} P_{m}(D) f(x) d x=\alpha(\infty)-\alpha\left(x_{1}\right) .
$$

TheOREM 24d. If $G(t) \in$ class III, if $\phi(t)$ is defined for $\left(t_{0}<t<\infty\right)$, if

$$
f(x)=\int_{-\infty}^{\infty} G(x-t) \phi(t) d t
$$

converges for some $x>t_{0}+b+\sum_{1}^{\infty} 1 / a_{k}$ and if $x_{1}$ is a point of continuity of $\phi(t)$, $x_{1}>t_{0}$, then

$$
\lim _{m \rightarrow \infty} P_{m}(D) f\left(x_{1}\right)=\phi\left(x_{1}\right) .
$$

The proofs of these theorems are essentially contained in the proofs given in Sections 18 and 19.

25. Relaxation of continuity conditions. We now return to the general kernel $G(t)$. It is natural to suppose from the known examples of our theory, for example, the Laplace and Stieltjes transforms, that if

$$
f(x)=\int_{-\infty}^{\infty} G(x-t) \phi(t) d t
$$

converges, then

$$
\lim _{m \rightarrow \infty} P_{m}(D) f(x)=\phi(x)
$$

almost everywhere. If the constants $b_{m}$ in the operator $E(D)$ which determine the magnitude of the arbitrary translations approach zero not too slowly, this may be shown to be true. For the Post-Widder operator $b_{m}$ $=O\left(m^{-1}\right), m \rightarrow \infty$, whereas the present theory would even permit $b_{m}=O\left(m^{-1 / 2}\right)$.

THEOREM 25a. If $G_{m}(t)$ is defined as in Theorem 3a, and if

$$
S_{m}=\sum_{m+1}^{\infty} \frac{1}{a_{k}^{2}},
$$

then

$$
G_{m}(t) \leqq 2^{1 / 2} S_{m}^{-1 / 2} \quad(-\infty<t<\infty) .
$$

There are two cases which we must consider.

A. $S_{m} \geqq 2 \max \left(\left|a_{m}+1\right|^{-2},\left|a_{m}+2\right|^{-2}, \cdots\right)$.

B. $S_{m}<2 \max \left(\left|a_{m}+1\right|^{-2},\left|a_{m}+2\right|^{-2}, \cdots\right)$.

Case A. We have

$$
G_{m}(t)=\frac{1}{2 \pi i} \int_{-i \infty}^{i \infty} \frac{e^{s t}}{e^{b_{m} s} \prod_{m+1}^{\infty}\left(1-\frac{s}{a_{k}}\right) e^{s / a_{k}}} d s,
$$




$$
G_{m}(t) \leqq \frac{1}{2 \pi} \int_{-\infty}^{\infty} \frac{d y}{\left[\prod_{m+1}^{\infty}\left(1+\frac{y^{2}}{a_{k}^{2}}\right)\right]^{1 / 2}}
$$

Now

$$
\prod_{m+1}^{\infty}\left(1+\frac{y^{2}}{a_{k}^{2}}\right)=1+y^{2} S_{m}+\frac{1}{2} y^{4} \sum_{k=m+1}^{\infty} \frac{1}{a_{k}^{2}}\left(S_{m}-\frac{1}{a_{k}^{2}}\right)+\cdots,
$$

the higher terms having positive coefficients. Since we are dealing with case A, we have

$$
S_{m}-\frac{1}{a_{k}^{2}} \geqq \frac{1}{2} S_{m}, \quad k=m+1, m+2, \cdots,
$$

and

$$
\prod_{m+1}^{\infty}\left(1+\frac{y^{2}}{a_{k}^{2}}\right) \geqq 1+y^{2} S_{m}+\frac{1}{4} y^{4} S_{m}^{2}
$$

Hence

$$
\begin{aligned}
G_{m}(t) & \leqq \frac{1}{2 \pi} \int_{-\infty}^{\infty} \frac{d y}{\left[1+y^{2} S_{m}+y^{4} S_{m}^{2} / 4\right]^{1 / 2}} \\
& \leqq \frac{1}{2 \pi} S_{m}^{-1 / 2} \int_{-\infty}^{\infty} \frac{d y}{1+y^{2} / 2} \\
& \leqq \frac{1}{2^{1 / 2}} S_{m}^{-1 / 2} .
\end{aligned}
$$

Case B. In this case there exists an integer $r$ greater than or equal to $m+1$, such that

$$
\frac{1}{a}>\frac{1}{2} S_{m}
$$

If we set

$$
R_{m}(t)=\frac{1}{2 \pi i} \int_{-i \infty}^{i \infty} \frac{e^{s t}}{e^{b_{m} s} \prod_{k=m+1, k \neq r}^{\infty}\left(1-\frac{s}{a_{k}}\right) e^{s / a_{k}}} d s,
$$

then we know from Theorem 12 that $R_{m}(t)$ is a frequency function. By the convolution theorem for the bilateral Laplace transform

$$
G_{m}(t)=\int_{-\infty}^{\infty} g_{r}(u) R_{m}(t-u) d u,
$$


where $g_{r}(u)$ is defined as in $\$ 1$. Now

$$
G_{m}(t) \leqq \max _{-\infty<u<\infty} g_{r}(u) \int_{-\infty}^{\infty} R_{m}(t-u) d u \leqq\left|a_{r}\right| .
$$

Since $\left|a_{r}\right|<2^{1 / 2} S_{m}^{-1 / 2}$ we obtain

$$
G_{m}(t) \leqq 2^{1 / 2} S_{m}^{-1 / 2} \quad(-\infty<t<\infty) .
$$

Combining the results for cases $\mathrm{A}$ and $\mathrm{B}$, we obtain our theorem. It is interesting to note that $S_{m}^{-1 / 2}$ is the true order of magnitude of $\max _{-\infty<t<\infty} G_{m}(t)$, since by (1), $\$ 13$

$$
\max _{-\infty<t<\infty} G_{m}(t) \geqq \frac{3}{16} S_{m}^{-1 / 2} .
$$

Theorem 25b. If in Theorems 19b, 23b, and 24d the assumption that $\phi(t)$ is continuous at $x_{1}$ is replaced by the weaker hypothesis

$$
\int_{x_{1}}^{x_{1}+h}\left[\phi(t)-\phi\left(x_{1}\right)\right] d t=o(h) \quad(h \rightarrow 0)
$$

and if it is assumed that $b_{m}=O\left(S_{m}^{1 / 2}\right)$, where $S_{m}$ is defined as in (1), then the conclusion of these theorems, that

$$
\lim _{m \rightarrow \infty} P_{m}(D) f\left(x_{1}\right)=\phi\left(x_{1}\right),
$$

still holds. In particular, equation (2) holds at all points of the Lebesgue set of $\phi(t)$ and therefore almost everywhere.

We must show that, given $\epsilon>0$, there exists $\delta>0$ such that if

$$
I_{m}=\int_{-\delta}^{\delta} G_{m}(t)\left[\phi\left(x_{1}-t\right)-\phi\left(x_{1}\right)\right] d t
$$

then

$$
\underset{m \rightarrow \infty}{\lim \sup _{m}}\left|I_{m}\right| \leqq \epsilon .
$$

If $\xi_{m}$ is the point associated with the unique change of sign of $G_{m}^{\prime}(t)$, then by Theorem 13b

$$
\left|\xi_{m}\right| \leqq\left|b_{m}\right|+8 S_{m}^{1 / 2},
$$

and hence

$$
\left|\xi_{m}\right|=O\left(S_{m}^{1 / 2}\right)
$$

Let 


$$
M=\sup _{m=1,2, \ldots}\left[\xi_{m} G\left(\xi_{m}\right)\right]
$$

This is finite by virtue of Theorem $25 \mathrm{a}$. We now choose $\delta$ so small that

$$
|\psi(t)| \leqq \frac{\epsilon}{2 M+1}|t| \quad(-\delta \leqq t \leqq \delta),
$$

where

$$
\psi(t)=\int_{0}^{t}\left[\phi\left(x_{1}-u\right)-\phi\left(x_{1}\right)\right] d u .
$$

Integrating by parts, and using Theorem $13 \mathrm{~b}$, we have

$$
\begin{aligned}
I_{m} & =\left[G_{m}(t) \psi(t)\right]_{-\delta}^{\delta}-\int_{-\delta}^{\delta} G_{m}^{\prime}(t) \psi(t) d t \\
\left|I_{m}\right| & \leqq o(1)+\frac{\epsilon}{2 M+1} \int_{-\delta}^{\delta}\left|G_{m}^{\prime}(t) t\right| d t \\
& \leqq o(1)+\frac{\epsilon}{2 M+1}\left[\int_{-\delta}^{\delta} G_{m}^{\prime}(t)\left(\xi_{m}-t\right) d t+\int_{-\delta}^{\delta}\left|\xi_{m} G_{m}^{\prime}(t)\right| d t\right] .
\end{aligned}
$$

Now

$$
\begin{aligned}
\int_{-\delta}^{\delta} G_{m}^{\prime}(t)\left(\xi_{m}-t\right) d t & =\left[G_{m}(t)\left(\xi_{m}-t\right)\right]_{-\delta}^{\delta}+\int_{-\delta}^{\delta} G_{m}(t) d t \\
& =1+o(1) \quad(m \rightarrow \infty), \\
\int_{-\delta}^{\delta}\left|\xi_{m} G_{m}^{\prime}(t)\right| d t & \leqq\left|\xi_{m}\right| \int_{-\infty}^{\xi_{m}} G_{m}^{\prime}(t) d t-\left|\xi_{m}\right| \int_{\xi_{m}}^{\infty} G_{m}^{\prime}(t) d t \\
& \leqq 2\left|\xi_{m}\right| G_{m}\left(\xi_{m}\right) \\
& \leqq 2 M .
\end{aligned}
$$

Thus finally

$$
\begin{gathered}
\left|I_{m}\right| \leqq o(1)+\frac{\epsilon}{2 M+1}[1+o(1)+2 M] \\
\limsup _{m \rightarrow \infty} I_{m} \leqq \epsilon
\end{gathered}
$$

Another result which was to be anticipated is that if $\alpha(t)$ is normalized,

$$
\alpha(t)=[\alpha(t+)+\alpha(t-)] / 2 \quad(-\infty<t<\infty),
$$

and if the transform 


$$
f(x)=\int_{-\infty}^{\infty} G(x-t) d \alpha(t)
$$

converges, then

$$
\lim _{m \rightarrow \infty} \int_{x_{1}}^{x_{2}} P_{m}(D) f(x) d x=\alpha\left(x_{2}\right)-\alpha\left(x_{1}\right)
$$

for all $x_{1}$ and $x_{2}$. This result is not unconditionally true, as we shall show by an example. However, we can give a simple sufficient condition which in the case of the Laplace transform at last yields as much information as the best known result (see Pollard [8]). The present result is sufficiently general to include all iterates of the Laplace transform.

TheOREM 25c. Let $G(t)$ be defined as in Theorem $3 a$ and let $S_{m}$ be defined as in Theorem 25a. If

$$
C_{m}=\sum_{m+1}^{\infty} \frac{1}{\left|a_{k}\right|^{3}}=o\left(S_{m}^{3 / 2}\right), \quad b_{m}=o\left(S_{m}^{1 / 2}\right),
$$

then

$$
\lim _{m \rightarrow \infty} \int_{-\infty}^{0} G_{m}(t) d t=\frac{1}{2}, \quad \lim _{m \rightarrow \infty} \int_{0}^{\infty} G_{m}(t) d t=\frac{1}{2} .
$$

Since

$$
\lim _{m \rightarrow \infty} \int_{-1}^{1} G_{m}(t) d t=1
$$

it will be enough to show that $\lim _{m \rightarrow \infty} \delta_{m}=0$, where

$$
\delta_{m}=\int_{0}^{1} G_{m}(t) d t-\int_{-1}^{0} G_{m}(t) d t .
$$

A short computation yields

$$
\begin{aligned}
\delta_{m} & =\frac{i}{\pi} \int_{-\infty}^{\infty} \frac{1-\cos y}{y E_{m}(i y)} d y \\
& =\frac{i}{\pi} \int_{0}^{\infty} \frac{1-\cos y}{y}\left[\frac{1}{E_{m}(i y)}-\frac{1}{E_{m}(-i y)}\right] d y .
\end{aligned}
$$

As $m \rightarrow \infty$ the function $E_{m}(s)$ is approaching 1 uniformly for $s$ in any bounded set. Hence

$$
\lim _{m \rightarrow \infty} \frac{i}{\pi} \int_{0}^{1} \frac{1-\cos y}{y}\left[\frac{1}{E_{m}(i y)}-\frac{1}{E_{m}(-i y)}\right] d y=0 .
$$


Thus it will be enough to show that, under our assumptions,

$$
\lim _{m \rightarrow \infty} \int_{1}^{\infty} \frac{1}{y}\left|\frac{1}{E_{m}(i y)}-\frac{1}{E_{m}(-i y)}\right| d y=0 .
$$

We split this integral into two parts $I_{m}$ and $J_{m}$ corresponding respectively to the ranges $\left(1, N S_{m}^{-1 / 2}\right)$ and $\left(N S_{m}^{-1 / 2}, \infty\right)$ where $N$ is an, as yet, unspecified positive constant. Since $E_{m}(i y)^{-1}$ and $E_{m}(-i y)^{-1}$ are conjugate

$$
\begin{aligned}
\left|\frac{1}{E_{m}(i y)}-\frac{1}{E_{m}(i y)}\right| & \leqq 2\left|\frac{1}{E_{m}(i y)}\right| \cdot\left|\sin \arg \frac{1}{E_{m}(i y)}\right| \\
& \leqq 2\left|\frac{1}{E_{m}(i y)}\right| \arg \left|\frac{1}{E_{m}(i y)}\right|
\end{aligned}
$$

Now $\left|1 / E_{m}(i y)\right| \leqq 1$. Furthermore

$$
\left|\arg \frac{1}{E_{m}(i y)}\right| \leqq\left|b_{m} y\right|+\sum_{k=m+1}^{\infty}\left|\frac{y}{a_{k}}-\tan ^{-1} \frac{y}{a_{k}}\right| .
$$

By Taylor's theorem with remainder

$$
\tan ^{-1} x=x-x^{3} \theta\left[1+(\theta x)^{2}\right]^{-1} \quad(0 x<\theta<1) .
$$

Hence

$$
\left|\frac{y}{a_{k}}-\tan ^{-1} \frac{y}{a_{k}}\right| \leqq \frac{y^{3}}{\left|a_{k}\right|^{3}}, \quad k=m+1, m+2, \cdots,
$$

and

$$
\begin{aligned}
I_{m} & \leqq 2 \int_{1}^{N S_{m}^{-1 / 2}} \frac{1}{y}\left[C_{m} y^{3}+\left|b_{m}\right| y\right] d y \\
& \leqq \frac{2}{3} N^{3} S_{m}^{-3 / 2} C_{m}+2 N S_{m}^{-1 / 2}\left|b_{m}\right|
\end{aligned}
$$

It follows that $\lim _{m \rightarrow \infty} I_{m}=0$. On the other hand, using (1), we see that

$$
\begin{aligned}
J_{m} & \leqq \int_{N S_{m}^{-1 / 2}}^{\infty} \frac{1}{y}\left[\left|\frac{1}{E_{m}(i y)}\right|+\left|\frac{1}{E_{m}(-i y)}\right|\right] d y \\
& \leqq \int_{N S_{m}^{-1 / 2}}^{\infty} \frac{2}{y} \frac{1}{\left[1+S_{m} y^{2}\right]^{1 / 2}} d y \\
& \leqq \int_{N}^{\infty} \frac{2}{-y} \frac{1}{\left[1+y^{2}\right]^{1 / 2}} d y .
\end{aligned}
$$


Thus

$$
\limsup _{m \rightarrow \infty}\left|\delta_{m}\right| \leqq \int_{N}^{\infty} \frac{2}{y} \frac{1}{\left[1+y^{2}\right]^{1 / 2}} d y .
$$

Since $N$ is arbitrary, our theorem follows.

ThEOREM 25d. If in Theorems 19a, 23a, and 24c, we make the additional assumptions that

$$
C_{m}=o\left(S_{m}^{3 / 2}\right), \quad b_{m}=o\left(S_{m}^{1 / 2}\right) \quad(m \rightarrow \infty)
$$

where $C_{m}$ and $S_{m}$ are defined as in the present section, then the conclusions of these theorems hold for all $x_{1}$ and $x_{2}$.

We leave the proof of this result to the reader.

We now turn to the construction of the example mentioned in the remarks preliminary to Theorem $25 \mathrm{c}$.

ThEOREM 25e. If $G_{m}(t)$ is defined as in Theorem 3a, if $b_{m}=0$, and if

$$
\sum_{m+2}^{\infty} \frac{1}{a_{k}^{2}} \leqq \frac{1}{10^{4} a_{m+1}^{2}}
$$

then

$$
\begin{array}{ll}
\int_{-\infty}^{0} G_{m}(t) d t \leqq .48 & \left(\text { if } a_{m+1}>0\right), \\
\int_{-\infty}^{0} G_{m}(t) d t \geqq .52 & \left(\text { if } a_{m+1}<0\right) .
\end{array}
$$

Since $\int_{-\infty}^{\infty} G_{m}(t) d t=1$, it will be sufficient to consider the case $a_{m+1}<0$. We have from (2), $\$ 1$, that

$$
G_{m}(t)=\int_{-\infty}^{\infty} g_{m+1}(u) R_{m+1}(t-u) d u,
$$

where

$$
\begin{array}{ll}
g_{m+1}(u)=-a_{m+1} e^{a_{m+1} t-1} & \left(t>a_{m+1}^{-1}\right), \\
g_{m+1}(u)=0 & \left(t<a_{m+1}^{-1}\right),
\end{array}
$$

and

$$
R_{m+1}(t)=\frac{1}{2 \pi i} \int_{-i \infty}^{i \infty} \frac{e^{s t}}{\prod_{m+2}^{\infty}\left(1-\frac{s}{a_{k}}\right) e^{s / a_{k}}} d s .
$$

By Theorem 12, $R_{m+1}(t)$ is a frequency function with variance $\sum_{m+2}^{\infty} a_{\mathrm{k}}^{-2}$ and 
mean 0 . By the argument used in the proof of Theorem $13 \mathrm{~b}$,

$$
\int_{-\delta}^{\delta} R_{m+1}(t) d t \geqq 1-\frac{I}{\delta^{2}} \sum_{m+2}^{\infty} \frac{1}{a_{k}^{2}} \geqq 1-.04=.96,
$$

if $\delta=\left(-20 a_{m+1}\right)^{-1}$ and inequality (3) is assumed. Now

$$
\begin{array}{rlrl}
G_{m}(t) & \geqq \min _{|u-t| \leqq \delta} g_{m+1}(u) \int_{t-\delta}^{t+\delta} R_{m}(t-u) d u \\
& \geqq-.96 a_{m+1} e^{a_{m+1}(t+\delta)-1} & \left(t \leqq a_{m+1}^{-1}+\delta\right) .
\end{array}
$$

Hence if $t_{0}=a_{m+1}^{-1}+\delta=.95 a_{m+1}^{-1}$, then

$$
\begin{aligned}
\int_{-\infty}^{0} G_{m}(t) d t & \geqq-.96 a_{m+1} e^{-1.05} \int_{t_{0}}^{0} e^{a_{m}+1 t} d t \\
& \geqq .96 e^{-1.05} \int_{0}^{.95} e^{y} d y \geqq .52
\end{aligned}
$$

which proves our theorem.

If we set

$$
E(s)=\prod_{1}^{\infty}\left(1-\frac{s}{e^{5 k}}\right),
$$

then it is easily verified that for every $m$

$$
\sum_{m+2}^{\infty} \frac{1}{e^{10 k}} \leqq \frac{1}{10^{4} e^{10(m+1)}} .
$$

Consequently no condition of the form

$$
b_{m}=O[w(m)]
$$$$
(m \rightarrow \infty),
$$

where $w(m)$ is a function of $m$, can insure that

$$
\lim _{m \rightarrow \infty} \int_{-\infty}^{0} G_{m}(t) d t=\lim _{m \rightarrow \infty} \int_{0}^{\infty} G_{m}(t) d t=\frac{1}{2}
$$

26. Generalization of the preceding theory. We wish now to generalize our theory in two ways. We shall permit $E(s)$ to have a zero of arbitrary order at the origin and in the line integral defining $G(s)$ we shall permit the line of integration to be (instead of the imaginary axis) any vertical line not passing through a root of $E(s)$. Let us begin with two preliminary results.

LEMMA 26a. If

$$
E^{*}(s)=s^{r} e^{b s} \prod_{1}^{\infty}\left(1-\frac{s}{a_{k}}\right) e^{s / a_{k}}
$$


then for any real number $c$ not a zero of $E^{*}(s)$

$$
E^{*}(s+c)=E^{*}(c) J(s),
$$

where $J(s)$ has the same type expansion as $E^{*}(s)$ :

$$
\begin{aligned}
J(s) & =e^{d s}\left(1+\frac{s}{c}\right)^{r} e^{-s r / c} \prod_{k=1}^{\infty}\left(1-\frac{s}{a_{k}-c}\right) e^{s /\left(a_{k}-c\right)}, \\
d & =b+\frac{r}{c}-\sum_{1}^{\infty} \frac{c}{a_{k}\left(a_{k}-c\right)} .
\end{aligned}
$$

Observe that $J(s)$ has for its zeros those of $E^{*}(s)$ each decreased by $c$ and that $J(0) \neq 0$. The proof of the lemma follows immediately from the algebraic identities

$$
\begin{gathered}
\left(1-\frac{c}{a_{k}}\right)\left(1-\frac{s}{a_{k}-c}\right)=1-\frac{s+c}{a_{k}}, \\
\frac{1}{a_{k}-c}-\frac{c}{a_{k}\left(a_{k}-c\right)}=\frac{1}{a_{k}} .
\end{gathered}
$$

LEMMA 26b. If $E^{*}(s), J(s)$ and $c$ are defined as in Lemma 26a, then

$$
J(D)\left[e^{-c x} f(x)\right]=\frac{e^{-c x}}{E^{*}(c)} E^{*}(D) f(x) .
$$

Here, as always, $J(D)$ and $E^{*}(D)$ are to be interpreted as limits, corresponding to the limits defining the infinite products involved. The proof is easily supplied by use of the differential identities

$$
\begin{aligned}
\left(1+\frac{D}{c}\right)\left[e^{-c x} f(x)\right] & =\frac{e^{-c x}}{c} D f(x), \\
\left(1-\frac{D}{a_{k}-c}\right)\left[e^{-c x} f(x)\right] & =\frac{a_{k} e^{-c x}}{a_{k}-c}\left(1-\frac{D}{a_{k}}\right) f(x) .
\end{aligned}
$$

THEOREM 26c. If

1. $E^{*}(s)=e^{b s} s^{r} \stackrel{\infty}{\prod}\left(1-\frac{s}{a_{k}}\right) e^{s / a_{k}}$

2. $G^{*}(t)=\frac{1}{2 \pi i} \int_{c-i \infty}^{c+i \infty} \frac{e^{s t}}{E^{*}(s)} d s \quad\left(c \neq a_{k}, k=1,2, \cdots ; c \neq 0\right)$,

3. $f(x)=\int_{-\infty}^{\infty} G^{*}(x-t) \phi(t) d t$

then 


$$
E^{*}(D) f(x)=\phi(x)
$$

in the same sense as in Theorems $19 \mathrm{~b}, 23 \mathrm{~b}$, and $24 \mathrm{~d}$.

By the change of variable $s=z+c$ we obtain

$$
G^{*}(t)=\frac{1}{2 \pi i} \int_{-i \infty}^{i \infty} \frac{e^{t(z+c)}}{E^{*}(z+c)} d z .
$$

By Lemma 26a, this becomes

$$
G^{*}(t)=\frac{e^{c t}}{2 \pi i} \int_{-i \infty}^{i \infty} \frac{e^{2 t}}{E^{*}(c) J(z)} d z .
$$

If we set

$$
H(t)=\frac{1}{2 \pi i} \int_{-i \infty}^{i \infty} \frac{e^{z t}}{J(z)} d z,
$$

we see that $H(t)$ is precisely the type of kernel $(J(0) \neq 0, c=0)$ that was studied throughout this paper. Moreover, the transform defined in condition 3 now becomes

$$
f(x) e^{-c x}=\int_{-\infty}^{\infty} H(x-t)\left[\frac{e^{-c t} \phi(t)}{E^{*}(c)}\right] d t .
$$

Hence it can be inverted by the operator $J(D)$,

$$
J(D)\left[f(x) e^{-c x}\right]=\frac{e^{-c x} \phi(x)}{E^{*}(c)} .
$$

But by Lemma 26b,

$$
J(D)\left[f(x) e^{-c x}\right]=\frac{e^{-c x}}{E^{*}(c)} E^{*}(D) f(x) .
$$

Hence

$$
E^{*}(D) f(x)=\phi(x)
$$

and our result is proved.

\section{BIBLIOGRAPHY}

1. E. J. Akutowicz, The third iterate of the Laplace transform, Duke Math. J. vol. 15(1948) pp. 1093-1132.

2. L. Bieberbach, Lehrbuch der Funktionentheorie, Leipzig, 1931.

3. R. P. Boas, Jr., Asymptotic relations for derivatives, Duke Math. J. vol. 3 (1937) pp. 637646.

4. - Inversion of a generalized Laplace integral, Proc. Nat. Acad. Sci. U.S.A. vol. 28 (1942) pp. 21-24. 
5. R. P. Boas, Jr., and D. V. Widder, The iterated Stieltjes transform, Trans. Amer. Math. Soc. vol. 45 (1939) pp. 1-72.

6. H. Cramér, Mathematical methods of statistics, Princeton, 1946.

7. I. I. Hirschman, Jr., and D. V. Widder, The inversion of convolution transforms with totally positive kernels, Proc. Nat. Acad. Sci. U.S.A. vol. 34 (1948) pp. 152-156.

8. Harry Pollard, Note on the inversion of the Laplace integral, Duke Math. J. vol. 6 (1940) pp. $420-424$.

9. — Integral transforms, Duke Math. J. vol. 13 (1946) pp. 307-330.

10. - The inversion of the transforms with reiterated Stieltjes kernels, Duke Math. J. vol. 14 (1947) pp. 129-142.

11. - The integral transforms with iterated Laplace kernels, Duke Math. J. vol. 14 (1947) pp. 659-675.

12. G. P6lya and G. Szegö, Aufgaben und Lehrsätze aus der Analysis, Berlin, 1925.

13. I. J. Schoenberg, On totally positive functions, Laplace integrals, and entire functions of the Laguerre-Pólya-Schur type, Proc. Nat. Acad. Sci. U.S.A. vol. 33 (1947) pp. 11-17.

14. D. V. Widder, Inversion of the Laplace integral and the related moment problem, Trans. Amer. Math. Soc. vol. 36 (1934) pp. 107-200.

15. - The Stieltjes transform, Trans. Amer. Math. Soc. vol. 43 (1937) pp. 7-60.

16. - The Green's function for a differential system of infinite order, Proc. Nat. Acad. Sci. U.S.A. vol. 26 (1940) pp. 213-215.

17. — The Laplace transform, Princeton, 1941.

18. - Inversion formulas for convolution transforms, Duke Math. J. vol. 14 (1947) pp. 217-251.

19. - The inversion of a generalized Laplace transform, Proc. Nat. Acad. Sci. U.S.A. vol. 33 (1947) pp. 295-297.

HaRVARD UNIVERSITY,

Cambridge, Mass. 